\title{
OVERLAPPING SCHWARZ METHODS FOR ISOGEOMETRIC ANALYSIS*
}

\author{
L. BEIRÃO DA VEIGA ${ }^{\dagger}$, D. CHO ${ }^{\ddagger}$, L. F. PAVARINO ${ }^{\dagger}$, AND S. SCACCHI $^{\dagger}$
}

\begin{abstract}
We construct and analyze an overlapping Schwarz preconditioner for elliptic problems discretized with isogeometric analysis. The preconditioner is based on partitioning the domain of the problem into overlapping subdomains, solving local isogeometric problems on these subdomains, and solving an additional coarse isogeometric problem associated with the subdomain mesh. We develop an $h$-analysis of the preconditioner, showing in particular that the resulting algorithm is scalable and its convergence rate depends linearly on the ratio between subdomain and "overlap sizes" for fixed polynomial degree $p$ and regularity $k$ of the basis functions. Numerical results in two- and three-dimensional tests show the good convergence properties of the preconditioner with respect to the isogeometric discretization parameters $h, p, k$, number of subdomains $N$, overlap size, and also jumps in the coefficients of the elliptic operator.
\end{abstract}

Key words. domain decomposition methods, overlapping Schwarz, scalable preconditioners, isogeometric analysis, finite elements, NURBS

AMS subject classifications. 65N55, 65N30, 65F 10

DOI. $10.1137 / 110833476$

1. Introduction. Isogeometric analysis (IGA) based on NURBS (nonuniform rational B-splines) was introduced by Hughes, Cottrell, and Bazilevs in [27] as an innovative numerical methodology for the analysis of PDE problems, allowing for an exact description of CAD-type geometries. NURBS are a standard in the computeraided design $(\mathrm{CAD})$ community mainly because such spline functions allow excellent representations of free-form surfaces, and there are very efficient algorithms for their evaluation, refinement, and derefinement. In the isogeometric framework, the NURBS basis functions representing the CAD geometry are also used as the basis for the discrete solution space of PDEs, following an isoparametric paradigm. In addition to exact representation of CAD geometries, another advantage of using NURBS basis functions is the higher control on the regularity of the discrete space. For instance, spaces of global $C^{k}$ regularity are easily built, thus allowing for fewer degrees of freedom, better performance in case of vibrations, easier approximation of higher order problems, and other advantages. IGA methodologies have been summarized in the recent book [18] and studied in, e.g., [2, 4, 9, 10, 19, 23, 28, 29, 11, 5, 8]. IGA methods are having a growing impact on fields as diverse as fluid dynamics $[6,7,40,15,26]$, structural mechanics $[3,1,12,20,30,39]$, and electromagnetics $[17,16]$.

The discrete systems produced by isogeometric methods are better conditioned than the systems produced by standard finite elements or finite differences, but their conditioning can still degenerates rapidly for decreasing mesh size $h$, increasing poly-

*Received by the editors May 10, 2011; accepted for publication (in revised form) January 16, 2012; published electronically May 31, 2012. This work was partially supported by FIRB grant RBFR08CZ0S.

http://www.siam.org/journals/sinum/50-3/83347.html

${ }^{\dagger}$ Dipartimento di Matematica, Università di Milano, Via Saldini 50, 20133 Milano, Italy (lourenco. beirao@unimi.it, luca.pavarino@unimi.it, simone.scacchi@unimi.it).

${ }^{\ddagger}$ Dipartimento di Matematica, Università di Milano, Via Saldini 50, 20133 Milano, Italy, and Department of Mathematics, Dongguk University, Pil-dong 3-ga, Jung-gu, Seoul, 100-715, South Korea (durkbin@imati.cnr.it). This author's work was done at the Department of Mathematics, University of Milan, where facilities for research were provided. 
nomial degrees $p$, and increasing coefficient jumps in the elliptic operator. Therefore the design and analysis of efficient iterative solvers for IGA is a challenging research topic, particularly for three-dimensional problems with discontinuous coefficients.

In the present paper we consider the following model elliptic problem:

$$
-\nabla \cdot(\rho \nabla u)=f \text { in } \Omega, \quad u=0 \text { on } \partial \Omega,
$$

where the scalar field $0<\rho_{\min } \leq \rho(x) \leq \rho_{\max }$ for all $x \in \Omega$ and $\Omega \subset \mathbb{R}^{d}$ is a bounded and connected CAD domain.

We construct and study overlapping Schwarz preconditioners for model elliptic problems (1.1), discretized by NURBS-based IGA. In our $h$-analysis, we prove that the resulting solvers are scalable (independent of the number of subdomains $N$ ) and have an optimal convergence rate depending linearly on the ratio $H / \gamma$ between the subdomain and overlap sizes. Since the underlying NURBS basis functions are not interpolatory, one cannot follow the standard proof used in finite elements, but must build ad-hoc spline operators that are stable and with local image. The results of extensive two-dimensional (2D) and three-dimensional (3D) numerical tests confirm our convergence rate bounds and additionally explore the condition number dependence on $p$ and $k$, showing that, as for standard spectral element Schwarz preconditioners, we have optimality in $p$ for generous overlap, for both minimal and maximal regularity $k$, while the growth in $p$ becomes stronger for minimal overlap and in particular for maximal regularity $k=p-1$. These tests show that the above good convergence properties also hold for heterogeneous problems with discontinuous coefficients across subdomain boundaries. Therefore, our isogeometric Schwarz preconditioners retain the good convergence properties of overlapping Schwarz solvers for standard Galerkin discretizations, for both $h$-finite elements (see, e.g., the books [37, 38]) and $h p$ or spectral elements (see, e.g., [32, 33, 25]). Our 2-level additive algorithm and analysis for IGA can be easily extended to 2-level multiplicative and hybrid versions, as in the finite and spectral element cases. We expect that the proposed 2-level overlapping Schwarz theory can also be extended toward elliptic systems such as compressible elasticity, while the extension to multilevel methods will need further research.

The paper is organized as follows. In section 2, we give a brief review of B-splines, NURBS, and IGA. In section 3, we present the overlapping Schwarz preconditioner and a bound of its condition number. The main part of the proof is then developed in section 4 , where we show the stable splitting property of the local discrete spaces. Finally, several numerical 2D and 3D tests are shown in section 5, confirming the theoretical convergence rate bounds.

2. NURBS-based isogeometric analysis. Nonuniform rational B-splines are a standard tool for describing and modeling curves and surfaces in CAD and computer graphics (see Piegl and Tiller [34] and Rogers [35] for an extensive description of these functions and their properties). In this work, we use NURBS as an analysis tool, as proposed by Hughes, Cottrell, and Bazilevs [27]. In this section, we present a short description of B-splines, NURBS, the basics of IGA, and an introduction to the proposed discretization method.

In the rest of the paper, we will adopt the following compact notation. Given two real numbers $a, b$ we write $a \lesssim b$ when $a \leq C b$ for a generic constant $C$ independent of the knot vectors (defined below), and we write $a \approx b$ when $a \lesssim b$ and $b \lesssim a$.

2.1. B-splines. B-splines in the plane are piecewise polynomial curves composed of linear combinations of B-spline basis functions. A knot vector is a set of non- 
decreasing real numbers representing coordinates in the parametric space of the curve

$$
\left\{\xi_{1}=0, \ldots, \xi_{n+p+1}=1\right\},
$$

where $p$ is the polynomial degree of the B-spline and $n$ is the number of basis functions (and control points) necessary to describe it. The interval $\left(\xi_{1}, \xi_{n+p+1}\right)$ is called a patch. A knot vector is said to be uniform if its knots are uniformly spaced and nonuniform otherwise. The maximum allowed knot multiplicity is $p+1$; a knot vector is said to be open if its first and last knots have multiplicity $p+1$. In what follows, we always employ open knot vectors. Basis functions formed from open knot vectors are interpolatory at the ends of the parametric interval $\widehat{\Omega}:=(0,1)$ but are not, in general, interpolatory at interior knots.

Given a knot vector, univariate B-spline basis functions are defined recursively starting with $p=0$ (piecewise constants):

$$
N_{i}^{0}(\xi)=\left\{\begin{array}{l}
1 \text { if } \xi_{i} \leq \xi<\xi_{i+1}, \\
0 \text { otherwise }
\end{array}\right.
$$

For $p>1$,

$$
N_{i}^{p}(\xi)= \begin{cases}\frac{\xi-\xi_{i}}{\xi_{i+p}-\xi_{i}} N_{i}^{p-1}(\xi)+\frac{\xi_{i+p+1}-\xi}{\xi_{i+p+1}-\xi_{i+1}} N_{i+1}^{p-1}(\xi) & \text { if } \xi_{i} \leq \xi<\xi_{i+p+1} \\ 0 & \text { otherwise }\end{cases}
$$

where, in (2.3), we adopt the convention $0 / 0=0$. Thus, the general basis function $N_{i}^{p}$ has support

$$
\Theta_{i}:=\operatorname{supp}\left(N_{i}^{p}\right)=\left(\xi_{i}, \xi_{i+p+1}\right), \quad i=1,2, \ldots, n .
$$

The functions $N_{i}^{p}$ are a partition of unity, as shown in [36]. In Figure 3.1 we present an example consisting of $n=9$ cubic basis functions generated from the open knot vector $\boldsymbol{\xi}=\{0,0,0,0,1 / 6,1 / 3,1 / 2,2 / 3,5 / 6,1,1,1,1\}$. If internal knots are not repeated, Bspline basis functions are $C^{p-1}$-continuous. If a knot has multiplicity $\alpha$, the basis is $C^{k}$-continuous, with $k=p-\alpha$, at that knot. In particular, when a knot has multiplicity $\alpha=p$, the basis is $C^{0}$ and interpolates the control point at that location. In the following, we will assume that the maximum knot multiplicity is $p$ so that all considered functions will be (at least) globally continuous. We define the spline space

$$
\widehat{\mathcal{S}}_{h}=\operatorname{span}\left\{N_{i}^{p}(\xi), i=1, \ldots, n\right\} .
$$

Following [36, Theorem 4.41], we define suitable functionals $\lambda_{j}^{p}=\lambda\left[\xi_{j}, \ldots, \xi_{j+p+1}\right]$, for $1 \leq j \leq n$, which are dual to the B-splines basis functions. Let

$$
y_{i}=\cos \left(\frac{p+1-i}{p+1}\right) \pi, \quad i=0,1, \ldots, p+1,
$$

and let

$$
B_{p+1}^{*}(x)=(p+1)(-1)^{p+1}\left[y_{0}, y_{1}, \ldots, y_{p+1}\right](\max \{x-y, 0\})^{p},
$$

where $\left[y_{0}, y_{1}, \ldots, y_{p+1}\right] f$ denotes the classical divided difference. We introduce a transition function $g$ defined as follows:

$$
g(x)=\left\{\begin{array}{cc}
0, & x<-1 \\
\int_{-1}^{x} B_{p+1}^{*}(t) d t, & -1 \leq x<1 \\
1, & 1 \leq x
\end{array}\right.
$$


(see [36, Theorem 4.37] for details). Let $G_{j}^{p}(x)=g\left(\frac{2 x-\xi_{j}-\xi_{j+p+1}}{\xi_{j+p+1}-\xi_{j}}\right)$ and $\phi_{j}^{p}(x)=$ $\frac{\left(x-\xi_{j+1}\right) \cdots\left(x-\xi_{j+p}\right)}{p !}$; then for $j=1, \ldots, n$, we set

$$
\lambda_{j}^{p}(f)=\int_{\xi_{j}}^{\xi_{j+p+1}} f(x) D^{p+1} \psi_{j}^{p}(x) d x \quad \forall f \in L^{2}(0,1),
$$

where $\psi_{j}^{p}(x)=G_{j}^{p}(x) \phi_{j}^{p}(x)$. The functionals $\lambda_{j}^{p}$ are constructed to verify

$$
\lambda_{j}^{p}\left(N_{i}^{p}\right)=\delta_{i j}, \quad 1 \leq i, j \leq n,
$$

where $\delta_{i j}$ is the Kronecker delta. The following estimate of the functional $\lambda_{j}^{p}$ defined in (2.5) will be useful in what follows.

Lemma 2.1. If $f \in L^{q}\left(\xi_{j}, \xi_{j+p+1}\right)$, with $1 \leq q \leq+\infty$, then

$$
\left|\lambda_{j}^{p}(f)\right| \lesssim\left|\xi_{j+p+1}-\xi_{j}\right|^{-1 / q}\|f\|_{L^{q}\left(\xi_{j}, \xi_{j+p+1}\right)} .
$$

The proof can be found in [36, Theorem 4.41]. Let $\triangle_{i}=\xi_{i+p}-\xi_{i}$ for $1 \leq$ $i \leq n$. We recall the formula for the derivative of univariate B-splines (see [36, Theorem 4.16]):

$$
\frac{d}{d \xi} N_{i}^{p}=p\left(\frac{N_{i}^{p-1}}{\triangle_{i}}-\frac{N_{i+1}^{p-1}}{\triangle_{i+1}}\right) .
$$

By means of tensor products, a multidimensional B-spline region can be constructed. We discuss here the case of a $2 \mathrm{D}$ region, the higher-dimensional case being analogous. Let $\widehat{\Omega}:=(0,1) \times(0,1)$ be the $2 \mathrm{D}$ parametric space. Consider the knot vectors $\left\{\xi_{1}=0, \ldots, \xi_{n+p+1}=1\right\}$ and $\left\{\eta_{1}=0, \ldots, \eta_{m+q+1}=1\right\}$, and an $n \times m$ net of control points $\mathbf{C}_{i, j}$. One-dimensional (1D) basis functions $N_{i}^{p}$ and $M_{j}^{q}$ (with $i=1, \ldots, n$ and $j=1, \ldots, m$ ) of degree $p$ and $q$, respectively, are defined from the knot vectors. The bivariate spline basis on $\widehat{\Omega}$ is then defined by the tensor product construction

$$
B_{i, j}^{p, q}(\xi, \eta)=N_{i}^{p}(\xi) M_{j}^{q}(\eta) .
$$

Observe that the two knot vectors $\left\{\xi_{1}=0, \ldots, \xi_{n+p+1}=1\right\}$ and $\left\{\eta_{1}=0, \ldots, \eta_{m+q+1}\right.$ $=1\}$ generate a mesh of rectangular elements in the parametric space in a natural way. Analogous to (2.4), we define

$$
\widehat{\mathcal{S}}_{h}=\operatorname{span}\left\{B_{i, j}^{p, q}(\xi, \eta), i=1, \ldots, n, j=1, \ldots, m\right\}
$$

2.2. NURBS maps and spaces. In general, a rational B-spline in $\mathbb{R}^{d}$ is the projection onto d-dimensional physical space of a polynomial B-spline defined in $(\mathrm{d}+1)$-dimensional homogeneous coordinate space. For a complete discussion, see the book by Farin [24] and the references therein. In this way, a great variety of geometrical entities can be constructed and, in particular, all conic sections in physical space can be obtained exactly. To obtain a NURBS curve in $\mathbb{R}^{2}$, we start introducing the NURBS basis functions of degree $p$,

$$
R_{i}^{p}(\xi)=\frac{N_{i}^{p}(\xi) \omega_{i}}{\sum_{\hat{i}=1}^{n} N_{\hat{i}}^{p}(\xi) \omega_{\hat{i}}}=\frac{N_{i}^{p}(\xi) \omega_{i}}{w(\xi, \eta)}
$$

where the denominator $w(\xi)=\sum_{\hat{i}=1}^{n} N_{\hat{i}}^{p}(\xi) \omega_{\hat{i}} \in \widehat{\mathcal{S}}_{h}$ is called the weight function. 
The NURBS curve is then defined by

$$
\mathbf{C}(\xi)=\sum_{i=1}^{n} R_{i}^{p}(\xi) \mathbf{C}_{i}
$$

where $\mathbf{C}_{i} \in \mathbb{R}^{2}$ are control points.

Analogously to B-splines, NURBS basis functions on the 2D parametric space $\widehat{\Omega}=(0,1) \times(0,1)$ are defined as

$$
R_{i, j}^{p, q}(\xi, \eta)=\frac{B_{i, j}^{p, q}(\xi, \eta) \omega_{i, j}}{\sum_{\hat{i}=1}^{n} \sum_{\hat{j}=1}^{m} B_{\hat{i}, \hat{j}}^{p, q}(\xi, \eta) \omega_{\hat{i}, \hat{j}}}=\frac{B_{i, j}^{p, q}(\xi, \eta) \omega_{i, j}}{w(\xi, \eta)},
$$

where $\omega_{i, j}=\left(\mathbf{C}_{i, j}^{\omega}\right)_{3}$ and the denominator is the weight function denoted also by $w(\xi, \eta)$. Observe that the continuity and support of NURBS basis functions are the same as for B-splines. NURBS spaces are the span of the basis functions (2.11).

NURBS regions are defined in terms of the basis functions (2.11). In particular a single-patch domain $\Omega$ is a NURBS region associated with the $n \times m$ net of control points $\mathbf{C}_{i, j}$, and we introduce the geometrical map $\mathbf{F}: \widehat{\Omega} \rightarrow \Omega$ given by

$$
\mathbf{F}(\xi, \eta)=\sum_{i=1}^{n} \sum_{j=1}^{m} R_{i, j}^{p, q}(\xi, \eta) \mathbf{C}_{i, j} .
$$

Following the isoparametric approach, the space of NURBS scalar fields on the domain $\Omega$ is defined, component by component, as the span of the push-forward of the basis functions (2.11),

$$
\mathcal{N}_{h}:=\operatorname{span}\left\{R_{i, j}^{p, q} \circ \mathbf{F}^{-1} \text {, with } i=1, \ldots, n ; j=1, \ldots, m\right\} .
$$

The image of the elements in the parametric space are elements in the physical space. The physical mesh on $\Omega$ is therefore

$$
\mathcal{T}_{h}=\left\{\mathbf{F}\left(\left(\xi_{i}, \xi_{i+1}\right) \times\left(\eta_{j}, \eta_{j+1}\right)\right), \text { with } i=1, \ldots, n+p, j=1, \ldots, m+q\right\},
$$

where the empty elements are not considered.

We are now able to present the isogeometric approximation of our model problem (1.1). As observed, for instance, in [4], in order to obtain spaces with homogeneous Dirichlet boundary conditions it is sufficient to eliminate the first and last functions in each coordinate. We therefore introduce the spline space (for instance, in two dimensions) living in parameter space,

$$
\widehat{V}=\widehat{\mathcal{S}}_{h} \cap H_{0}^{1}(\widehat{\Omega})=\operatorname{span}\left\{B_{i, j}^{p, q}(\xi, \eta), i=2, \ldots, n-1, j=2, \ldots, m-1\right\},
$$

and the NURBS space living in physical space,

$$
V=\mathcal{N}_{h} \cap H_{0}^{1}(\Omega)=\operatorname{span}\left\{R_{i, j}^{p, q} \circ \mathbf{F}^{-1}, \text { with } i=2, \ldots, n-1 ; j=2, \ldots, m-1\right\} .
$$

We are now able to write the discrete formulation of the model problem (1.1): Find $u \in V$ such that

$$
a(u, v)=\int_{\Omega} f v d x \quad \forall v \in V,
$$

with bilinear form $a(u, v)=\int_{\Omega} \rho \nabla u \nabla v d x$ and $0<\rho_{\min } \leq \rho(x) \leq \rho_{\max }$ for all $x \in \Omega$. 
The interested reader may find more details on IGA as well as many interesting applications in a number of recently published papers mentioned in the introduction. The $3 \mathrm{D}$ case is analogous and not discussed.

Remark 2.1. Throughout the paper we consider Dirichlet homogeneous boundary conditions only for simplicity. Different kinds of boundary conditions can be clearly treated in the same fashion.

3. The overlapping Schwarz preconditioner. In this section, we construct the isogeometric overlapping Schwarz preconditioner and present a theoretical bound on the condition number of the preconditioned operator. We refer the reader to, e.g., $[37,38,22]$ for a general introduction to Overlapping Schwarz methods.

3.1. Subdomains and subspaces. We describe first the subdomain and subspace decompositions in one dimension and then extend them by tensor products to two and three dimensions. The decomposition is first built for the underlying space of spline functions in parameter space, and then easily extended to the NURBS space in the physical domain. We select from the full set of knots $\left\{\xi_{1}=0, \ldots, \xi_{n+p+1}=1\right\}$ a subset $\left\{\xi_{i_{k}}, k=1, \ldots, N+1\right\}$ of (nonrepeated) interface knots with $\xi_{i_{1}}=0, \xi_{i_{N+1}}=1$. This subset of interface knots defines a decomposition of the closure of the reference interval

$$
\overline{(\widehat{I})}=[0,1]=\overline{\left(\bigcup_{k=1, \ldots, N} \widehat{I}_{k}\right)} \text {, with } \widehat{I}_{k}=\left(\xi_{i_{k}}, \xi_{i_{k+1}}\right)
$$

which we assume to have a similar characteristic diameter $H \approx H_{k}=\operatorname{diam}\left(\widehat{I}_{k}\right)$. The interface knots are thus given by $\xi_{i_{k}}$ for $k=2, \ldots, N$. For each of the interface knots $\xi_{i_{k}}$ we choose an index $2 \leq s_{k} \leq n-1$ (strictly increasing in $k$ ) that satisfies $s_{k}<i_{k}<s_{k}+p+1$, so that the support of the basis function $N_{s_{k}}^{p}$ intersects both $\widehat{I}_{k-1}$ and $\widehat{I}_{k}$. Note that at least one such $s_{k}$ exists; if it is not unique, any choice can be taken.

We then define an overlapping decomposition of $\widehat{I}$ in the following way. Let $r \in \mathbb{N}$ be an integer (called the overlap index) counting the basis functions shared by adjacent subdomains, defined as

$$
\widehat{V}_{k}=\operatorname{span}\left\{N_{j}^{p}(\xi), s_{k}-r \leq j \leq s_{k+1}+r\right\}, \quad k=1,2, \ldots, N,
$$

with the exception that $2 \leq j \leq s_{2}+r$ for the space $\widehat{V}_{1}$ and $s_{N}-r \leq j \leq n-1$ for the space $\widehat{V}_{N}$ (an example is shown in Figure 3.1). These subspaces form an overlapping decomposition of the spline space $\widehat{V}$. For $r=0$ we have the minimal overlap consisting of just one common basis function between subspaces. We also define the overlap parameter

$$
\gamma=h(2 r+2) .
$$

Note that the overlap parameter $\gamma$ is related to the width $\delta$ of the overlapping region by the bounds

$$
\gamma=h(2 r+2) \leq \delta \leq h(2 r+p+1) \leq \frac{p+1}{2} \gamma,
$$

and also that $2 r+1$ represents the number of basis functions in common (in the univariate case) among "adjacent" local subspaces. We now define the extended 


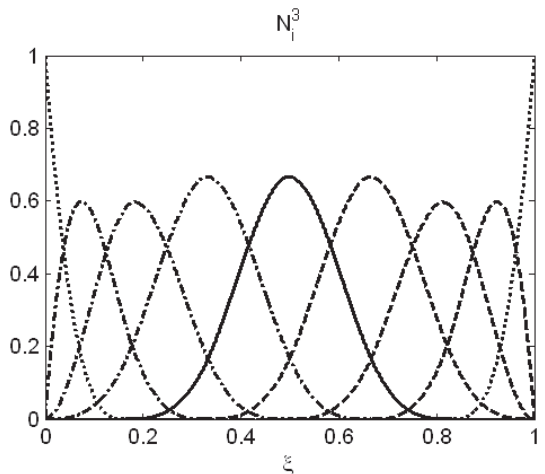

(a) $r=0$

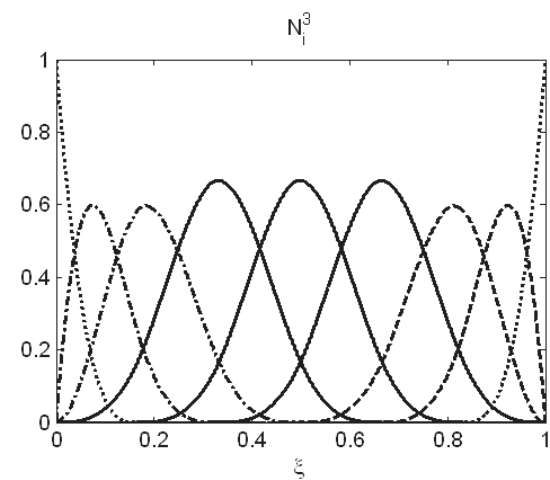

(b) $r=1$

Fig. 3.1. Cubic basis functions formed from $\boldsymbol{\xi}=\{0,0,0,0,1 / 6,1 / 3,1 / 2,2 / 3,5 / 6,1,1,1,1\}$. For various $r, \widehat{V}_{1}$ is the span of basis functions drawn with dash-dot and solid lines and $\widehat{V}_{2}$ is the span of basis functions drawn with solid and dashed lines, in two subdomains $\widehat{I}_{1}=(0,1 / 2)$ and $\widehat{I}_{2}=(1 / 2,1)$ of $\widehat{I}$. In particular, the basis functions in common are those drawn with a solid line.

subdomains $\widehat{I}_{k}^{\prime}$ by

$$
\widehat{I}_{k}^{\prime}=\bigcup_{N_{j}^{p} \in \widehat{V}_{k}} \operatorname{supp}\left(N_{j}^{p}\right)=\left(\xi_{s_{k}-r}, \xi_{s_{k+1}+r+p+1}\right)
$$

with the analogous exception for $\widehat{I}_{1}^{\prime}, \widehat{I}_{N}^{\prime}$, and the further extended subdomains $\widehat{I}_{k}^{\prime \prime}$

$$
\widehat{I}_{k}^{\prime \prime}=\bigcup_{\operatorname{supp}\left(N_{j}^{p}\right) \cap \widehat{I}_{k}^{\prime} \neq \emptyset} \operatorname{supp}\left(N_{j}^{p}\right) \text {. }
$$

Let us then introduce an (open) coarse knot vector $\boldsymbol{\xi}_{0}=\left\{\xi_{1}^{0}=0, \ldots, \xi_{N_{c}+p+1}^{0}=1\right\}$ corresponding to a coarse mesh determined by the subdomains $\widehat{I}_{k}$, i.e.,

$$
\boldsymbol{\xi}_{0}=\left\{\xi_{1}, \xi_{2}, \ldots, \xi_{p}, \xi_{i_{1}}, \xi_{i_{2}}, \xi_{i_{3}}, \ldots, \xi_{i_{N-1}}, \xi_{i_{N}}, \xi_{i_{N}+1}, \xi_{i_{N}+2} \ldots, \xi_{i_{N}+p+1}\right\},
$$

such that the distance between adjacent distinct knots is of order $H, \xi_{1}=\cdots=\xi_{p}=$ $\xi_{i_{1}}=0$, and $\xi_{i_{N+1}}=\xi_{i_{N}+2}=\cdots=\xi_{i_{N}+p+1}=1$, and the associated coarse spline space

$$
\widehat{V}_{0}:=\widehat{\mathcal{S}}_{H}=\operatorname{span}\left\{N_{i}^{0, p}(\xi), i=1, \ldots, N_{c}\right\}
$$

has the same degree $p$ of $\widehat{\mathcal{S}}_{h}$ and is thus a subspace of $\widehat{\mathcal{S}}_{h}$. Note that, in order to have a smaller coarse space $\widehat{V}_{0}$, in the definition above we repeat only once all the internal knots. Nevertheless, less regular coarse spaces can be considered by including more repetitions of the interior knots.

In more than one dimension, we just proceed by tensor product. For example, in two dimensions we define subdomains, overlapping subdomains, and extended supports by

$$
\begin{aligned}
& \widehat{I}_{k}=\left(\xi_{i_{k}}, \xi_{i_{k+1}}\right), \quad \widehat{I}_{l}=\left(\eta_{j_{l}}, \eta_{j_{l+1}}\right), \quad \widehat{\Omega}_{k l}=\widehat{I}_{k} \times \widehat{I}_{l}, \\
& \widehat{\Omega}_{k l}^{\prime}=\widehat{I}_{k}^{\prime} \times \widehat{I}_{l}^{\prime}, \quad \widehat{\Omega}_{k l}^{\prime \prime}=\widehat{I}_{k}^{\prime \prime} \times \widehat{I}_{l}^{\prime \prime}, \quad 1 \leq k \leq N, 1 \leq l \leq M \text {. }
\end{aligned}
$$

Copyright $@$ ㅇ by SIAM. Unauthorized reproduction of this article is prohibited. 
Moreover, we take the indexes $\left\{s_{k}\right\}_{k=2}^{N}$ associated to the $\left\{\xi_{i_{k}}\right\}_{k=2}^{N}$ and the analogous indexes $\left\{\bar{s}_{l}\right\}_{l=2}^{M}$ associated to the $\left\{\eta_{j_{l}}\right\}_{l=2}^{M}$. The local and coarse subspaces are then defined by

$$
\begin{aligned}
\widehat{V}_{k l} & =\operatorname{span}\left\{B_{i, j}^{p, q}(\xi, \eta), s_{k}-r \leq i \leq s_{k+1}+r, \bar{s}_{l}-r \leq j \leq \bar{s}_{l+1}+r\right\}, \\
\widehat{V}_{0} & =\operatorname{span}\left\{B_{i, j}^{p, q}: B_{i, j}^{p, q}(\xi, \eta):=N_{i}^{0, p}(\xi) M_{j}^{0, q}(\eta), i=1, \ldots, N_{c}, j=1, \ldots, M_{c}\right\},
\end{aligned}
$$

with the usual modification for boundary subdomains and where $\stackrel{\circ}{B}_{i, j}^{p, q}$ are the coarse basis functions.

The decomposition for the NURBS space $V$ in the physical domain is the trivial extension of the one above. Therefore the local subspaces and the coarse space are, up to the usual modification for the boundary subdomains,

$$
\begin{aligned}
V_{k l} & =\operatorname{span}\left\{R_{i, j}^{p, q} \circ \mathbf{F}^{-1}, s_{k}-r \leq i \leq s_{k+1}+r, \bar{s}_{l}-r \leq j \leq \bar{s}_{l+1}+r\right\}, \\
V_{0} & =\operatorname{span}\left\{\stackrel{\circ}{R}_{i, j}^{p, q} \circ \mathbf{F}^{-1}:=\left(\stackrel{\circ}{B}_{i, j}^{p, q} / w\right) \circ \mathbf{F}^{-1}, i=1, \ldots, N_{c}, j=1, \ldots, M_{c}\right\},
\end{aligned}
$$

where we recall that $w$ is the weight function; see (2.11). The subdomains in physical space are defined as the image of the subdomains in parameter space with respect to the mapping $\mathbf{F}$,

$$
\Omega_{k l}=\mathbf{F}\left(\widehat{\Omega}_{k l}\right), \quad \Omega_{k l}^{\prime}=\mathbf{F}\left(\widehat{\Omega}_{k l}^{\prime}\right), \quad \Omega_{k l}^{\prime \prime}=\mathbf{F}\left(\widehat{\Omega}_{k l}^{\prime \prime}\right)
$$

We are now able to present the preconditioner Schwarz operator. Given the embedding operators $\mathbf{I}_{k l}: V_{k l} \rightarrow V, k=1, \ldots, N, l=1, \ldots, M \mathbf{I}_{0}: V_{0} \rightarrow V$, we define the projections $\widetilde{\mathbf{T}}_{k l}: V \rightarrow V_{k l}, \widetilde{\mathbf{T}}_{0}: V \rightarrow V_{0}$ by

$$
a\left(\widetilde{\mathbf{T}}_{k l} u, v\right)=a\left(u, \mathbf{I}_{k l} v\right) \quad \forall v \in V_{k l}, \quad a\left(\widetilde{\mathbf{T}}_{0} u, v\right)=a\left(u, \mathbf{I}_{0} v\right) \quad \forall v \in V_{0},
$$

and $\mathbf{T}_{k l}=\mathbf{I}_{k l} \widetilde{\mathbf{T}}_{k l}, \mathbf{T}_{0}=\mathbf{I}_{0} \widetilde{\mathbf{T}}_{0}$. Our 2-level overlapping additive Schwarz (OAS) operator is then

$$
\mathbf{T}_{O A S}:=\mathbf{T}_{0}+\sum_{k=1}^{N} \sum_{l=1}^{M} \mathbf{T}_{k l}
$$

The matrix form of this operator is

$$
\mathbf{T}_{O A S}=\mathbf{B}_{O A S} \mathcal{A},
$$

where $\mathcal{A}$ is the stiffness matrix and $\mathbf{B}_{O A S}$ is the additive Schwarz preconditioner:

$$
\mathbf{B}_{O A S}=R_{0}^{T} A_{0}^{-1} R_{0}+\sum_{k=1}^{N} \sum_{l=1}^{M} R_{k l}^{T} A_{k l}^{-1} R_{k l} .
$$

Here, $R_{k l}$ are restriction matrices with 0,1 entries returning the coefficients of the basis functions belonging to the local spaces $V_{k l}$, and $A_{k l}$ are the local stiffness matrices restricted to the subspace $V_{k l}$. $R_{0}$ is the fine-to-coarse restriction matrix, and $A_{0}$ is the coarse stiffness matrix associated with the coarse space $V_{0}$. The number of rows of $R_{0}$ is the dimension of the fine space, while the number of columns is the dimension of the coarse space. Each column of $R_{0}$ contains the coefficients of a coarse space basis function expressed as linear combination of the fine space basis functions.

More general multiplicative and hybrid versions as well as projection-like operators $\widetilde{\mathbf{T}}_{k l}$ associated with inexact local solvers could also be used (see [37, 38]) but will not be considered here. The use of the $\mathbf{B}_{O A S}$ preconditioner (3.7) for the iterative 
solution of the discrete system $\mathcal{A} \mathbf{u}=\mathbf{f}$ can also be regarded as replacing it with the preconditioned system

$$
\mathbf{T}_{O A S} \mathbf{u}=\mathbf{g}
$$

where $\mathbf{g}=\mathbf{B}_{O A S} \mathbf{f}$, which can be accelerated by a Krylov subspace method. In the next sections, we will prove a convergence rate bound for the condition number of $\mathbf{T}_{O A S}$. The $3 \mathrm{D}$ case is handled analogously.

Apart from the preprocessing stage of assembling the isogeometric discrete problem, the computational costs of our preconditioned iterative solver, analogously to the finite and spectral element cases, are dominated by solving the local problems on the overlapping subdomains and solving the coarse problem. The remaining restriction, interpolation, and vector operations have lower costs. A complexity analysis of this preconditioner for scalar elliptic problems and finite elements can be found in the book [37], for both serial (Chapter 3.6.1) and parallel computers (Chapter 3.6.3). A more detailed analysis of the parallel complexity of the 2-level overlapping Schwarz algorithm can be found in the book [31], where in sections 5.2.3 and 5.2.4 the total parallel time and parallel efficiency of the overlapping Schwarz preconditioner are explicitly estimated. Extending these complexity estimates to the isogeometric case is beyond the scope of this paper.

3.2. Condition number bounds. We make the following two assumptions on the mesh and subdivision. The first one is a standard assumption also in the finite element literature (see [38]), while the second one allows us to focus on the case of main interest in applications, i.e., when the overlap region is not excessive with respect to the mesh size:

(a) the parametric mesh in each extended subdomain $\widehat{\Omega}_{k l}^{\prime \prime}$ is uniform, i.e., there exists a real number $h=h\left(\widehat{\Omega}_{k l}^{\prime \prime}\right)$ such that all elements $E$ in $\widehat{\Omega}_{k l}^{\prime \prime}$ have a diameter which is equivalent to $h$ up to a constant that is fixed and which is the same for all subdomains;

(b) the overlap index $r$ is bounded from above by a fixed constant.

The following theorem is the main theoretical result of this contribution.

THEOREM 3.1. The condition number of the 2-level additive Schwarz preconditioned isogeometric operator $\mathbf{T}_{O A S}$ defined in (3.6) is bounded by

$$
\kappa_{2}\left(\mathbf{T}_{O A S}\right) \leq C\left(1+\frac{H}{\gamma}\right)
$$

where $\gamma=h(2 r+2)$ is the overlap parameter defined in (3.2) and $C$ is a constant independent of $h, H, N, \gamma$ (but not of $p, k$ ).

Proof. The proof follows the general abstract Schwarz theory (see, e.g., [38, Chapter 2]), based on verifying three assumptions known as stable decomposition [38, Assumption 2.2], strengthened Cauchy-Schwarz inequality [38, Assumption 2.3] and local stability [38, Assumption 2.4], which provide an upper and lower bound on the extreme eigenvalues of $\mathbf{T}_{O A S}$; see [38, Theorem 2.7].

(i) Since we use exact local solvers, the local stability assumption holds true with a unit constant.

(ii) By using a standard coloring argument (see [38, Chapter 2.5.1]), we obtain that the strengthened Cauchy-Schwarz inequality holds with a constant bounded from above by the number of colors. In the simple case where the nonoverlapping subdomains $\Omega_{i j}$ form a structured Cartesian decomposition of the original domain and 
the associated overlapping subdomains have not too large an overlap, this constant is 4 in two dimensions and 8 in three dimensions.

(iii) We are then left with proving a stable decomposition for our subspace decomposition. Such a task will be done in the following section.

Remark 3.1. In the following, we will assume that the elliptic coefficient $\rho$ appearing in the bilinear form of $(2.15)$ is equal to 1 everywhere in $\Omega$. The theory for the general case follows analogously, but with the addition of a factor $\rho_{\max } / \rho_{\min }$ in front of the condition number bound. Nevertheless, the numerical tests of section 5 will show that in practice the condition number of the preconditioned system is essentially independent of the coefficient $\rho$.

4. Stable decomposition. In this section, we prove the stable decomposition result needed in the proof of Theorem 3.1. We start with some preliminary results for the univariate case which will be fundamental for the following analysis.

4.1. Initial results in one dimension. We define interpolation operators $\widehat{\Pi}^{k}$ : $\widehat{V} \rightarrow \widehat{V}_{k}$ needed in our stable splitting. Any $z \in \widehat{V}$ can be represented as a linear combination of $\left\{N_{i}^{p}\right\}_{i=2}^{n-1}$; i.e., there exist constants $c_{j}, 2 \leq j \leq n-1$ such that

$$
z=\sum_{j=2}^{n-1} c_{j} N_{j}^{p}
$$

We first focus our attention on the case with only two subdomains, since this case already contains all the difficulties involved in our analysis and the extension to the general case will be straightforward. Following the notation and construction of section 3.1 , we have $\widehat{I}_{1}=\left(0, \xi_{i_{2}}\right), \widehat{I}_{2}=\left(\xi_{i_{2}}, 1\right)$ and that the closure of $\widehat{I}$ is given by $[0,1]$, the union of the closures of $\widehat{I}_{1}$ and $\widehat{I}_{2}$. Moreover, the local spaces are

$$
\widehat{V}_{1}=\operatorname{span}\left\{N_{i}^{p}(\xi), 2 \leq i \leq s_{2}+r\right\}, \quad \widehat{V}_{2}=\operatorname{span}\left\{N_{i}^{p}(\xi), s_{2}-r \leq i \leq n-1\right\},
$$

with $r \geq 0$ and $s_{2}<i_{2}<s_{2}+p+1$. Using this classification, we can write $z$ as in (4.1) and introduce two interpolation operators $\widehat{\Pi}^{k}: \widehat{V} \rightarrow \widehat{V}_{k}, k=1,2$, defined as follows:

$$
z=\sum_{j=2}^{n-1} c_{j} N_{j}^{p}=\widehat{\Pi}^{1} z+\widehat{\Pi}^{2} z \in \widehat{V}_{1}+\widehat{V}_{2}
$$

such that

$$
\begin{aligned}
& \widehat{\Pi}^{1} z=\sum_{j=2}^{s_{2}-r-1} c_{j} N_{j}^{p}+\sum_{j=s_{2}-r}^{s_{2}+r} \frac{\left(s_{2}+r+1\right)-j}{2 r+2} c_{j} N_{j}^{p} \in \widehat{V}_{1}, \\
& \widehat{\Pi}^{2} z=\sum_{j=s_{2}-r}^{s_{2}+r} \frac{\left(r-s_{2}+1\right)+j}{2 r+2} c_{j} N_{j}^{p}+\sum_{j=s_{2}+r+1}^{n-1} c_{j} N_{j}^{p} \in \widehat{V}_{2} .
\end{aligned}
$$

For brevity, we rewrite $\widehat{\Pi}^{1} z=\sum_{j=2}^{s_{2}+r} \bar{c}_{j} N_{j}^{p}$, with coefficients

$$
\bar{c}_{j}=c_{j} d_{j}, \quad \text { where } \quad d_{j}=\left\{\begin{array}{cl}
1 & \text { if } 2 \leq j \leq s_{2}-r-1, \\
\frac{\left(s_{2}+r+1\right)-j}{2 r+2} & \text { if } s_{2}-r \leq j \leq s_{2}+r .
\end{array}\right.
$$

It is immediate to check from the definition that the identity $z=\widehat{\Pi}^{1} z+\widehat{\Pi}^{2} z$ holds. Moreover, we have the following result. 
LEMma 4.1. The operators $\widehat{\Pi}^{k}, k=1,2$, satisfy the following bounds for all $z \in \widehat{V}:$

$$
\begin{aligned}
\left\|\frac{d}{d \xi}\left(\widehat{\Pi}^{k} z\right)\right\|_{L^{2}\left(\widehat{I}_{k}^{\prime}\right)}^{2} & \lesssim\left(1+\frac{H}{\gamma}\right)\left\|\frac{d}{d \xi} z\right\|_{L^{2}\left(\widehat{I}_{k}^{\prime \prime}\right)}^{2}+\frac{1}{H \gamma}\|z\|_{L^{2}\left(\widehat{I}_{k}^{\prime \prime}\right)}^{2}, \\
\left\|\widehat{\Pi}^{k} z\right\|_{L^{2}\left(\widehat{I}_{k}^{\prime}\right)} & \lesssim\|z\|_{L^{2}\left(\widehat{I}_{k}^{\prime \prime}\right)}
\end{aligned}
$$

where $\widehat{I}_{k}^{\prime}$ and $\widehat{I}_{k}^{\prime \prime}$ have been defined in (3.4) and (3.5), respectively.

Proof. It is sufficient to prove the result only for $\widehat{\Pi}^{1}$, since the result for $\widehat{\Pi}^{2}$ follows from the same argument. We first show (4.5). By definition and the derivative formula (2.7), it is immediate to check that

$$
\frac{d}{d \xi}\left(\widehat{\Pi}^{1} z\right)=\sum_{j=2}^{s_{2}+r} \bar{c}_{j} \frac{d}{d \xi} N_{j}^{p}=p \sum_{j=2}^{s_{2}+r+1}\left(\bar{c}_{j}-\bar{c}_{j-1}\right) \frac{N_{j}^{p-1}}{\triangle_{j}}
$$

where we adopted the convention that $\bar{c}_{1}=\bar{c}_{s_{2}+r+1}=0$. Given $\bar{c}_{j}$ as in (4.4), an easy computation shows that for all $\xi$ in $\widehat{I}$

$$
\begin{aligned}
\frac{d}{d \xi}\left(\widehat{\Pi}^{1} z\right)(\xi) & =p \sum_{j=2}^{s_{2}+r+1}\left[c_{j}\left(d_{j}-d_{j-1}\right)\right] \frac{N_{j}^{p-1}(\xi)}{\triangle_{j}}+p \sum_{j=2}^{s_{2}+r+1}\left[d_{j-1}\left(c_{j}-c_{j-1}\right)\right] \frac{N_{j}^{p-1}(\xi)}{\triangle_{j}} \\
& :=0
\end{aligned}
$$

We will estimate the terms $T_{1}$ and $T_{2}$ separately. Note that

$$
d_{j}-d_{j-1}=\left\{\begin{array}{cl}
0 & \text { if } 2 \leq j \leq s_{2}-r-1, \\
-\theta & \text { if } j \geq s_{2}-r
\end{array}\right.
$$

with $\theta=\frac{1}{2 r+2}$. Due to assumption (a) at the beginning of section 3.2, we have $\triangle_{j} \geq h$; hence for all $\xi \in \widehat{I}_{1}^{\prime}$ it holds that

$$
\left|T_{1}(\xi)\right| \lesssim \frac{1}{h} \sum_{s_{2}-r}^{s_{2}+r+1} \theta\left|c_{j}\right| N_{j}^{p-1} .
$$

Due to property (2.6) of the dual basis and the definition of $z=\sum_{j=2}^{n-1} c_{j} N_{j}^{p}$, it holds that $c_{j}=\lambda_{j}^{p}(z)$. Therefore, using Lemma 2.1 with $q=+\infty$ yields for all $s_{2}-r \leq j \leq s_{2}+r+1$

$$
\left|c_{j}\right|=\left|\lambda_{j}^{p}(z)\right| \lesssim \|\left. z\right|_{L^{\infty}\left(\operatorname{supp}\left(T_{1}\right)\right)} .
$$

This bound, together with (4.7) and the partition of unity property of the spline basis functions, gives

$$
\left|T_{1}(\xi)\right| \lesssim \frac{\theta}{h} \max _{s_{2}-r \leq j \leq s_{2}+r+1}\left|c_{j}\right| \lesssim \frac{\theta}{h}\|z\|_{L^{\infty}\left(\operatorname{supp}\left(T_{1}\right)\right)} .
$$

We now note that the support of $T_{1}$ satisfies $\operatorname{supp}\left(T_{1}\right):=\bigcup_{j=s_{2}-r}^{s_{2}+r+1} \operatorname{supp}\left(N_{j}^{p-1}\right)=$ $\left(\xi_{s_{2}-r}, \xi_{s_{2}+r+p+1}\right)$. Thus, squaring both sides in (4.8) and integrating over $\widehat{I}_{1}^{\prime}$, one 
gets

$$
\begin{aligned}
\int_{\widehat{I}_{1}^{\prime}}\left|T_{1}(\xi)\right|^{2} \mathrm{~d} \xi & =\int_{\operatorname{supp}\left(T_{1}\right)}\left|T_{1}(\xi)\right|^{2} \mathrm{~d} \xi \lesssim\left|\xi_{s_{2}+r+p+1}-\xi_{s_{2}-r}\right|\left\|T_{1}\right\|_{L^{\infty}\left(\operatorname{supp}\left(T_{1}\right)\right)}^{2} \\
& \lesssim \frac{\theta^{2}}{h^{2}}\left|\xi_{s_{2}+r+p+1}-\xi_{s_{2}-r}\right|\|z\|_{L^{\infty}\left(\operatorname{supp}\left(T_{1}\right)\right)}^{2} .
\end{aligned}
$$

By (3.2) the overlap parameter is $\gamma=\frac{h}{\theta}=h(2 r+2)$ and by (3.3), we have $\mid \xi_{s_{2}+r+p+1}-$ $\xi_{s_{2}-r} \mid \lesssim(2 r+p+1) h \lesssim \gamma$, and thus we obtain

$$
\int_{\widehat{I}_{1}^{\prime}}\left|T_{1}(\xi)\right|^{2} \mathrm{~d} \xi \lesssim \gamma^{-1}\|z\|_{L^{\infty}\left(\operatorname{supp}\left(T_{1}\right)\right)}^{2} \lesssim \gamma^{-1}\|z\|_{L^{\infty}\left(\widehat{I}_{1}^{\prime \prime}\right)}^{2}
$$

We now combine a standard scaling argument with the $H^{1} \subset L^{\infty}$ 1D Sobolev embedding; such a bound, applied to the above inequality, gives

$$
\int_{\widehat{I}_{1}^{\prime}}\left|T_{1}(\xi)\right|^{2} \mathrm{~d} \xi \lesssim \frac{H}{\gamma}\left\|\frac{d}{d \xi} z\right\|_{L^{2}\left(\widehat{I}_{1}^{\prime \prime}\right)}^{2}+\frac{1}{H \gamma}\|z\|_{L^{2}\left(\widehat{I}_{1}^{\prime \prime}\right)}^{2} .
$$

We now bound the second term $T_{2}$. For any general $\varphi=\sum_{j=2}^{n} \beta_{j} N_{j}^{p-1}$, Lemma 2.1 with the choice $q=2$ gives

$$
\left|\beta_{j}\right|=\left|\lambda_{j}^{p-1}(\varphi)\right| \lesssim h^{-1 / 2}\|\varphi\|_{L^{2}\left(\xi_{j}, \xi_{j+p}\right)} .
$$

Since $z=\sum_{j=2}^{n-1} c_{j} N_{j}^{p}$, the derivative formula (2.7) yields

$$
\frac{d}{d \xi} z=p \sum_{j=2}^{n} \frac{c_{j}-c_{j-1}}{\triangle_{j}} N_{j}^{p-1}
$$

which combined with (4.10) gives

$$
p\left|\frac{c_{j}-c_{j-1}}{\triangle_{j}}\right| \lesssim h^{-1 / 2}\left\|\frac{d}{d \xi} z\right\|_{L^{2}\left(\xi_{j}, \xi_{j+p}\right)},
$$

where we adopted the convention that $c_{1}=c_{n}=0$. By the definition of $d_{j}, T_{2}$ can be rewritten as

$$
T_{2}=p \sum_{j=2}^{s_{2}-r}\left(c_{j}-c_{j-1}\right) \frac{N_{j}^{p-1}}{\triangle_{j}}+p \sum_{j=s_{2}-r+1}^{s_{2}+r+1}\left[d_{j-1}\left(c_{j}-c_{j-1}\right)\right] \frac{N_{j}^{p-1}}{\triangle_{j}} .
$$

This splitting implies that the restriction of $T_{2}$ to $\left(0, \xi_{s_{2}-r+1}\right)$ coincides with $\frac{d}{d \xi} z$, as can be noted by comparing with (4.11). Therefore

$$
\begin{aligned}
& \int_{\widehat{I}_{1}^{\prime}}\left|T_{2}(\xi)\right|^{2} \mathrm{~d} \xi=\int_{\left(0, \xi_{s_{2}-r+1}\right)}\left|T_{2}(\xi)\right|^{2} \mathrm{~d} \xi+\int_{\left(\xi_{s_{2}-r+1}, \xi_{s_{2}+r+p+1}\right)}\left|T_{2}(\xi)\right|^{2} \mathrm{~d} \xi \\
& =\int_{\left(0, \xi_{s_{2}-r+1}\right)}\left|\frac{d}{d \xi} z\right|^{2} \mathrm{~d} \xi+\int_{\left(\xi_{s_{2}-r+1}, \xi_{s_{2}+r+p+1}\right)}\left|T_{2}(\xi)\right|^{2} \mathrm{~d} \xi .
\end{aligned}
$$

Moreover, from the definition of $T_{2}$, the partition of unity property and bound (4.12), it follows for all $\xi \in \operatorname{supp}\left(T_{2}\right)$ that

$$
\left|T_{2}(\xi)\right| \lesssim \max _{2 \leq j \leq s_{2}+r+1} p\left|\frac{c_{j}-c_{j-1}}{\triangle_{j}}\right| \lesssim h^{-1 / 2}\left\|\frac{d}{d \xi} z\right\|_{L^{2}\left(\widehat{I}_{1}^{\prime \prime}\right)} .
$$

Copyright $($ C by SIAM. Unauthorized reproduction of this article is prohibited. 
First using Hölder's inequality followed by (4.14), then recalling the assumptions at the start of section 3.2 , from (4.13) we have

$$
\begin{aligned}
\int_{\widehat{I}_{1}^{\prime}}\left|T_{2}(\xi)\right|^{2} \mathrm{~d} \xi & \lesssim\left\|\frac{d}{d \xi} z\right\|_{L^{2}\left(\widehat{I}_{1}\right)}^{2}+\left|\xi_{s_{2}+r+p+1}-\xi_{s_{2}-r+1}\right|\left\|T_{2}\right\|_{L^{\infty}\left(\xi_{s_{2}-r+1}, \xi_{s_{2}+r+p+1}\right)}^{2} \\
& \lesssim\left\|\frac{d}{d \xi} z\right\|_{L^{2}\left(\widehat{I}_{1}\right)}^{2}+h^{-1}\left|\xi_{s_{2}+r+p+1}-\xi_{s_{2}-r+1}\right|\left\|\frac{d}{d \xi} z\right\|_{L^{2}\left(0, \xi_{s_{2}+r+p+1}\right)}^{2} \\
& \lesssim(2 r+p+1)\left\|\frac{d}{d \xi} z\right\|_{L^{2}\left(\widehat{I}_{1}^{\prime \prime}\right)}^{2} \lesssim\left\|\frac{d}{d \xi} z\right\|_{L^{2}\left(\widehat{I}_{1}^{\prime \prime}\right)}^{2} .
\end{aligned}
$$

Combining the above inequality with (4.9), the first bound (4.5) follows.

It remains to show (4.6). Let $e=\left(\xi_{m}, \xi_{m+1}\right) \subset \widehat{I}_{1}^{\prime}$ be any element of the mesh. Then by Lemma 2.1 with $q=2$, for all $\xi \in e$, we get

$$
\widehat{\Pi}^{1} z(\xi)=\sum_{j=m-p}^{m} \bar{c}_{j} N_{j}^{p}(\xi) \leq \max _{m-p \leq j \leq m}\left|c_{j}\right| \lesssim h^{-1 / 2}\|z\|_{L^{2}\left(\xi_{m-p}, \xi_{m+p+1}\right)},
$$

where we also used the obvious property that $\left|\bar{c}_{j}\right| \leq\left|c_{j}\right|$ for all values of $j$. Thus,

$$
\int_{e}\left|\widehat{\Pi}^{1} z\right|^{2} \lesssim h^{-1} h\|z\|_{L^{2}\left(\xi_{m-p}, \xi_{m+p+1}\right)}^{2}=\|z\|_{L^{2}\left(\xi_{m-p}, \xi_{m+p+1}\right)}^{2} .
$$

Summing over all elements in the mesh yields $\left\|\widehat{\Pi}^{1} z\right\|_{L^{2}\left(\widehat{I}_{1}^{\prime}\right)} \lesssim\|z\|_{L^{2}\left(\widehat{I}_{1}^{\prime \prime}\right)}$, completing the proof.

The general case with $N$ subdomains is straightforward. If $z=\sum_{j=2}^{n-1} c_{j} N_{j}^{p}$, then

$$
z=\sum_{k=1}^{N} \widehat{\Pi}^{k} z
$$

where the operators $\widehat{\Pi}^{k}: \widehat{V} \rightarrow \widehat{V}_{k}$ are defined by

$\widehat{\Pi}^{k} z=\sum_{j=s_{k}-r}^{s_{k}+r} \frac{\left(r-s_{k}+1\right)+j}{2 r+2} c_{j} N_{j}^{p}+\sum_{j=s_{k}+r+1}^{s_{k+1}-r-1} c_{j} N_{j}^{p}+\sum_{j=s_{k+1}-r}^{s_{k+1}+r} \frac{\left(s_{k+1}+r+1\right)-j}{2 r+2} c_{j} N_{j}^{p}$,

with the obvious modification for the two boundary subdomains. Note that above we are ruling out for simplicity the uninteresting case where the overlap index $r$ is so big that it induces an overlap of the indexes of nonadjacent subdomains (i.e., we are assuming $\left.s_{k}+r+1 \leq s_{k+1}-r-1\right)$.

Clearly, the stability bounds are also derived by a trivial extension of the arguments shown in the two-subdomain case. We thus have the following result.

THEOREM 4.1. The operators $\widehat{\Pi}^{k}, k=1,2, \ldots, N$, satisfy the following bounds for all $z \in \widehat{V}$ :

$$
\begin{aligned}
\left\|\frac{d}{d \xi}\left(\widehat{\Pi}^{k} z\right)\right\|_{L^{2}\left(\widehat{I}_{k}^{\prime}\right)}^{2} & \lesssim\left(1+\frac{H}{\gamma}\right)\left\|\frac{d}{d \xi} z\right\|_{L^{2}\left(\widehat{I}_{k}^{\prime \prime}\right)}^{2}+\frac{1}{H \gamma}\|z\|_{L^{2}\left(\widehat{I}_{k}^{\prime \prime}\right)}^{2}, \\
\left\|\widehat{\Pi}^{k} z\right\|_{L^{2}\left(\widehat{I}_{k}^{\prime}\right)} & \lesssim\|z\|_{L^{2}\left(\widehat{I}_{k}^{\prime \prime}\right)} .
\end{aligned}
$$

Copyright $@$ by SIAM. Unauthorized reproduction of this article is prohibited. 
4.2. Stable splitting for splines in parameter domain. In this section, we present the stable splitting result for the case of splines in the parametric domain. For simplicity, we present the result in the $2 \mathrm{D}$ case, as the $3 \mathrm{D}$ case is analogous.

Given any function $v$ in the bivariate spline space $\widehat{V}$, we define its restrictions $v_{\xi}:(0,1) \rightarrow \mathbb{R}$ and $v_{\eta}:(0,1) \rightarrow \mathbb{R}:$

$$
v_{\xi}(\eta)=v(\xi, \eta) \quad \text { and } \quad v_{\eta}(\xi)=v(\xi, \eta) \quad \forall(\xi, \eta) \in \widehat{\Omega} .
$$

The functions above are clearly in the univariate spline space of degree $p$ generated by the knot vectors $\boldsymbol{\xi}$ and $\boldsymbol{\eta}$, respectively. We build the needed operators by a tensor product construction. For all $k=1,2, \ldots, N$ and $l=1,2, \ldots, M$ we introduce the linear operators $\widehat{\Pi}_{\xi}^{k}$ and $\widehat{\Pi}_{\eta}^{l}$, from $\widehat{V}$ to $\widehat{V}$, defined by

$$
\left(\widehat{\Pi}_{\xi}^{k} v\right)(\xi, \eta)=\left(\widehat{\Pi}^{k} v_{\eta}\right)(\xi) \text { and }\left(\widehat{\Pi}_{\eta}^{l} v\right)(\xi, \eta)=\left(\widehat{\Pi}^{l} v_{\xi}\right)(\eta) \quad \forall(\xi, \eta) \in \widehat{\Omega}, \forall v \in \widehat{V},
$$

where the $\widehat{\Pi}^{k}$ (and $\widehat{\Pi}^{l}$ ) are the 1D operators introduced in the previous section. It is easy to check that, due to the tensor product nature of the space $\widehat{V}$ and its basis, the above operators commute, i.e., $\widehat{\Pi}_{\xi}^{k} \circ \widehat{\Pi}_{\eta}^{l}=\widehat{\Pi}_{\eta}^{l} \circ \widehat{\Pi}_{\xi}^{k}$. For the same reason, the operators commute also with the derivative in the other direction, i.e.,

$$
\frac{\partial}{\partial \xi}\left(\widehat{\Pi}_{\eta}^{l} v\right)=\widehat{\Pi}_{\eta}^{l}\left(\frac{\partial}{\partial \xi} v\right) \quad \text { and } \quad \frac{\partial}{\partial \eta}\left(\widehat{\Pi}_{\xi}^{k} v\right)=\widehat{\Pi}_{\xi}^{k}\left(\frac{\partial}{\partial \eta} v\right) \quad \forall v \in V
$$

and for all $k, l$ in the above ranges. We are now able to define the linear operators

$$
\begin{aligned}
& \widehat{\Pi}^{k l}: \widehat{V} \longrightarrow \widehat{V}_{k l}, \\
& \widehat{\Pi}^{k l} v=\widehat{\Pi}_{\xi}^{k} \circ \widehat{\Pi}_{\eta}^{l}(v)=\widehat{\Pi}_{\eta}^{l} \circ \widehat{\Pi}_{\xi}^{k}(v) \quad \forall v \in \widehat{V} .
\end{aligned}
$$

Let $\widehat{\Pi}^{0}$ be the standard spline quasi-interpolant into the space $\widehat{V}_{0}$, which is built using the dual basis functions as detailed in [36, Theorem 12.6]. Given any $\widehat{u} \in \widehat{V}$, define

$$
\widehat{z}=\widehat{u}-\widehat{u}_{0} \in \widehat{V}, \quad \widehat{u}_{0}=\widehat{\Pi}^{0} \widehat{u} \in \widehat{V}_{0} .
$$

Due to well-known stability and approximation properties of the quasi-interpolant in [36, Theorem 12.7], the following bound holds:

$$
\|\widehat{z}\|_{L^{2}(\widehat{\Omega})}^{2}+H^{2}\left\|\nabla \widehat{u}_{0}\right\|_{L^{2}(\widehat{\Omega})}^{2} \lesssim H^{2}\|\nabla \widehat{u}\|_{L^{2}(\widehat{\Omega})}^{2} .
$$

Using the linearity of the operators in (4.18) and the splitting property (4.15) of the $1 \mathrm{D}$ operators, we obtain

$$
\sum_{k=1}^{N} \sum_{l=1}^{M} \widehat{\Pi}^{k l} \widehat{z}=\sum_{k=1}^{N} \widehat{\Pi}_{\xi}^{k}\left(\sum_{l=1}^{M} \widehat{\Pi}_{\eta}^{l} \widehat{z}\right)=\sum_{k=1}^{N} \widehat{\Pi}_{\xi}^{k} \widehat{z}=\widehat{z} .
$$

Therefore, denoting $\widehat{u}_{k l}=\widehat{\Pi}^{k l} \widehat{z}$, we have the splitting

$$
\widehat{u}=\widehat{u}_{0}+\sum_{k=1}^{N} \sum_{l=1}^{M} \widehat{u}_{k l}, \quad \widehat{u}_{0} \in \widehat{V}_{0}, \widehat{u}_{k l} \in \widehat{V}_{k l} .
$$

We are left to show that the splitting is stable, which we formalize in the next result. 
Proposition 4.1. For all $\widehat{u} \in \widehat{V}$ it holds that

$$
\left\|\nabla \widehat{u}_{0}\right\|_{L^{2}(\widehat{\Omega})}^{2}+\sum_{k=1}^{N} \sum_{l=1}^{M}\left\|\nabla \widehat{u}_{k l}\right\|_{L^{2}(\widehat{\Omega})}^{2} \lesssim\left(1+\frac{H}{\gamma}\right)\|\nabla \widehat{u}\|_{L^{2}(\widehat{\Omega})}^{2} .
$$

Proof. The result for the $\widehat{u}_{0}$ follows immediately from (4.22). Regarding the second term, we treat only the derivative in $x$, the derivative in $y$ clearly following with the same arguments. Let any $1 \leq k \leq N$ and $1 \leq l \leq M$. Since by definition $\operatorname{supp}\left(\widehat{u}_{k l}\right)=\widehat{\Omega}_{k l}^{\prime}$, using definition (4.20) and property (4.19) yields

$$
\left\|\frac{\partial}{\partial \xi} \widehat{u}_{k l}\right\|_{L^{2}(\widehat{\Omega})}^{2}=\left\|\frac{\partial}{\partial \xi} \widehat{\Pi}_{\eta}^{l} \circ \widehat{\Pi}_{\xi}^{k} \widehat{z}\right\|_{L^{2}\left(\widehat{\Omega}_{k l}^{\prime}\right)}^{2}=\left\|\widehat{\Pi}_{\eta}^{l} \frac{\partial}{\partial \xi}\left(\widehat{\Pi}_{\xi}^{k} \widehat{z}\right)\right\|_{L^{2}\left(\widehat{\Omega}_{k l}^{\prime}\right)}^{2} .
$$

Now let $v$ be any function in $\widehat{V}$. Then, first by definition of $\widehat{\Omega}_{k l}^{\prime}$ and (4.18), and then using standard calculus and the 1D bound (4.17), we get

$$
\begin{aligned}
\left\|\widehat{\Pi}_{\eta}^{l} v\right\|_{L^{2}\left(\widehat{\Omega}_{k l}^{\prime}\right)}^{2} & =\int_{\widehat{I}_{k}^{\prime}} \int_{\widehat{I}_{l}^{\prime}}\left|\left(\widehat{\Pi}_{\eta}^{l} v\right)(\xi, \eta)\right|^{2} \mathrm{~d} \eta \mathrm{d} \xi=\int_{\widehat{I}_{k}^{\prime}} \int_{\widehat{I}_{l}^{\prime}}\left|\left(\widehat{\Pi}^{l} v_{\xi}\right)(\eta)\right|^{2} \mathrm{~d} \eta \mathrm{d} \xi \\
& \lesssim \int_{\widehat{I}_{k}^{\prime}} \int_{\widehat{I}_{l}^{\prime \prime}}\left|v_{\xi}(\eta)\right|^{2} \mathrm{~d} \eta \mathrm{d} \xi=\int_{\widehat{I}_{k}^{\prime}} \int_{\widehat{I}_{l}^{\prime \prime}}|v(\xi, \eta)|^{2} \mathrm{~d} \eta \mathrm{d} \xi .
\end{aligned}
$$

We now apply bound (4.27) to the identity (4.26). By standard calculus, definition (4.18), and using the $1 \mathrm{D}$ bound (4.16), we get

$$
\begin{aligned}
\left\|\frac{\partial}{\partial \xi} \widehat{u}_{k l}\right\|_{L^{2}(\widehat{\Omega})}^{2} & \lesssim \int_{\widehat{I}_{k}^{\prime}} \int_{\widehat{I}_{l^{\prime \prime}}}\left|\frac{\partial}{\partial \xi}\left(\widehat{\Pi}_{\xi}^{k} \widehat{z}\right)(\xi, \eta)\right|^{2} \mathrm{~d} \eta \mathrm{d} \xi=\int_{\widehat{I}_{l}^{\prime \prime}} \int_{\widehat{I}_{k}^{\prime}}\left|\frac{\partial}{\partial \xi}\left(\widehat{\Pi}^{k} \widehat{z}_{\eta}\right)(\xi)\right|^{2} \mathrm{~d} \xi \mathrm{d} \eta \\
& \lesssim\left(1+\frac{H}{\gamma}\right) \int_{\widehat{I}_{l}^{\prime \prime}} \int_{\widehat{I}_{k}^{\prime \prime}}\left|\frac{\partial}{\partial \xi} \widehat{z}_{\eta}(\xi)\right|^{2} \mathrm{~d} \xi \mathrm{d} \eta+\frac{1}{H \gamma} \int_{\widehat{I}_{l}^{\prime \prime}} \int_{\widehat{I}_{k}^{\prime \prime}}\left|\widehat{z}_{\eta}(\xi)\right|^{2} \mathrm{~d} \xi \mathrm{d} \eta \\
& =\left(1+\frac{H}{\gamma}\right)\left\|\frac{\partial}{\partial \xi} \widehat{z}\right\|_{L^{2}\left(\widehat{\Omega}_{k l}^{\prime \prime}\right)}^{2}+\frac{1}{H \gamma}\|\widehat{z}\|_{L^{2}\left(\widehat{\Omega}_{k l}^{\prime \prime}\right)}^{2} .
\end{aligned}
$$

Since the above bound holds for all $(k, l)$ and the number of overlaps of the extended subdomains $\widehat{\Omega}_{k l}^{\prime \prime}$ is uniformly bounded, by a standard coloring argument inequality (4.28) gives

$$
\sum_{k=1}^{N} \sum_{l=1}^{M}\left\|\frac{\partial}{\partial \xi} \widehat{u}_{k l}\right\|_{L^{2}(\widehat{\Omega})}^{2} \lesssim\left(1+\frac{H}{\gamma}\right)\left\|\frac{\partial}{\partial \xi} \widehat{z}\right\|_{L^{2}(\widehat{\Omega})}^{2}+\frac{1}{H \gamma}\|\widehat{z}\|_{L^{2}(\widehat{\Omega})}^{2} .
$$

Recalling the definition of $\widehat{\Omega}$ and using property (4.22), some simple algebra gives from (4.29)

$$
\sum_{k=1}^{N} \sum_{l=1}^{M}\left\|\frac{\partial}{\partial \xi} \widehat{u}_{k l}\right\|_{L^{2}(\widehat{\Omega})}^{2} \lesssim\left(1+\frac{H}{\gamma}\right)\|\nabla \widehat{u}\|_{L^{2}(\widehat{\Omega})}^{2} .
$$

The proof follows applying the same argument for the $\frac{\partial}{\partial \eta}$ derivative.

The proof of Theorem 3.1 for the case of the spline space in the parameter domain finally follows by combining Proposition 4.1 with the steps mentioned in section 3.2. The full isogeometric case, including map and weight, is considered in the next section. 
Remark 4.1. Note that the proof above is based on a tensor product argument, which allows us to use the $1 \mathrm{D}$ results of the previous section, considering one coordinate derivative at a time. Therefore, nothing changes in the case of the three (or actually arbitrary) dimensions, where the same proof applies. The more cumbersome notation required is the reason why we decided to focus the presentation on the $2 \mathrm{D}$ case.

4.3. Stable splitting for NURBS in physical domain. We now present the stable splitting results in the full isogeometric case, again for simplicity in two dimensions, as the 3D case is analogous. Let $u$ be any function in the mapped NURBS space $V$. By definition of the space $V$, it is immediate to check that

$$
V=\left\{\frac{\widehat{v}}{w} \circ \mathbf{F}^{-1}: \widehat{v} \in \widehat{V}\right\}
$$

where we recall that $w$ represents the weight function. As a consequence, there exists $\widehat{u} \in \widehat{V}$ such that $u=(\widehat{u} / w) \circ \mathbf{F}^{-1}$ and, conversely, $\widehat{u}=w(u \circ \mathbf{F})$. Using the results in the previous section, we have the existence of a stable splitting for $\widehat{u}$. Therefore, introducing the functions

$$
u_{k l}=\frac{\widehat{u}_{k l}}{w} \circ \mathbf{F}^{-1} \in V_{k l}, \quad u_{0}=\frac{\widehat{u}_{0}}{w} \circ \mathbf{F}^{-1} \in V_{0},
$$

we clearly have from (4.24) the splitting $u=u_{0}+\sum_{k=1}^{N} \sum_{l=1}^{M} u_{k l}$. We are left to prove that the above splitting in physical space is stable.

Proposition 4.2. For all $u \in V$ it holds that

$$
\left\|\nabla u_{0}\right\|_{L^{2}(\Omega)}^{2}+\sum_{k=1}^{N} \sum_{l=1}^{M}\left\|\nabla u_{k l}\right\|_{L^{2}(\Omega)}^{2} \lesssim\left(1+\frac{H}{\gamma}\right)\|\nabla u\|_{L^{2}(\Omega)}^{2} .
$$

Proof. The functions $w, \mathbf{F}$ are continuous, piecewise regular, and fixed once and for all at the coarsest level of discretization. We make, moreover, the standard assumption (see [4]) that the inverse mapping $\mathbf{F}^{-1}$ is well defined and with bounded derivatives. As a consequence, we have that $w, \mathbf{F}, \mathbf{F}^{-1}$ are (componentwise) in the $W^{1, \infty}$ Sobolev space. Therefore, first a standard change of variables (see, for instance, [4]) from $\Omega$ to $\widehat{\Omega}$, then a Poincaré inequality on $\widehat{\Omega}$ give

$$
\begin{aligned}
\left\|\nabla u_{0}\right\|_{L^{2}(\Omega)}^{2}+\sum_{k=1}^{N} \sum_{l=1}^{M}\left\|\nabla u_{k l}\right\|_{L^{2}(\Omega)}^{2} & \lesssim\left\|\widehat{u}_{0}\right\|_{H^{1}(\widehat{\Omega})}^{2}+\sum_{k=1}^{N} \sum_{l=1}^{M}\left\|\widehat{u}_{k l}\right\|_{H^{1}(\widehat{\Omega})}^{2} \\
& \lesssim\left\|\nabla \widehat{u}_{0}\right\|_{L^{2}(\widehat{\Omega})}^{2}+\sum_{k=1}^{N} \sum_{l=1}^{M}\left\|\nabla \widehat{u}_{k l}\right\|_{L^{2}(\widehat{\Omega})}^{2} .
\end{aligned}
$$

We now apply Proposition 4.1 in (4.32), map back into $\Omega$, and finally apply again a Poincaré inequality to obtain the thesis

$$
\begin{aligned}
& \left\|\nabla u_{0}\right\|_{L^{2}(\Omega)}^{2}+\sum_{k=1}^{N} \sum_{l=1}^{M}\left\|\nabla u_{k l}\right\|_{L^{2}(\Omega)}^{2} \\
& \quad \lesssim\left(1+\frac{H}{\gamma}\right)\|\nabla \widehat{u}\|_{L^{2}(\widehat{\Omega})}^{2} \\
& \quad \lesssim\left(1+\frac{H}{\gamma}\right)\|u\|_{H^{1}(\Omega)}^{2} \lesssim\left(1+\frac{H}{\gamma}\right)\|\nabla u\|_{L^{2}(\Omega)}^{2} .
\end{aligned}
$$

Copyright $@$ by SIAM. Unauthorized reproduction of this article is prohibited. 
The proof of Theorem 3.1 finally follows by combining Proposition 4.2 with the steps already mentioned. We underline again the full generality of the result with respect to the problem dimension.

Note finally that in the above argument the coarse space $\widehat{V}_{0}$ is not required to respect the isoparametric paradigm. Therefore, the coarse mesh defining the coarse space does not need to be a refinement of the coarse mesh which is used to define the geometry (i.e., $\mathbf{F}$ and $w$ ).

Remark 4.2. The arguments shown here always refer to the case of a single-patch geometry, but the extension to the multipatch case is straightforward. Indeed, the different patches can be used as an initial subdivision into subdomains (and possibly divided again). Since the standard multipatch structure involves a $C^{0}$ gluing of the discrete functions across different patches, this interfaces can be dealt with as in standard finite element overlapping Schwarz methods. The more difficult case is instead the one already treated in this contribution, i.e., the case when a higher $C^{k}$ regularity is present across the subdomain interfaces.

5. Numerical results. We now test the convergence properties of the isogeometric overlapping Schwarz preconditioner (3.7) for two-dimensions and threedimensions model elliptic problems (2.15) on both parametric (reference square or cube) and physical domains (see Figure 5.3), with zero right-hand side, Dirichlet boundary conditions $e^{x} \sin (y)$ in two dimensions or mixed boundary conditions in three dimensions. The problem is discretized with isogeometric NURBS spaces with associated mesh size $h$, polynomial degree $p$, regularity $k$, using the MATLAB isogeometric library GeoPDEs [21]. The domain is decomposed into $N$ overlapping subdomains of characteristic size $H$ and overlap index $r$, as described in section 3.1. The discrete problems are solved by the preconditioned conjugate gradient (PCG) method with isogeometric Schwarz preconditioner (3.7), with zero initial guess and as stopping criterion a $10^{-6}$ reduction of the relative PCG residual.

In the following tests, we study how the convergence rate of the overlapping additive Schwarz (OAS) preconditioner depends on $h, N, p, k$ and on the jumps of the elliptic coefficients.

5.1. 2D tests: OAS scalability in $\boldsymbol{N}$ and optimality in $\boldsymbol{H} / \boldsymbol{h}$. The condition number $\kappa_{2}\left(T_{O A S}\right)$ and iteration counts of the OAS preconditioner are reported in Table 5.1 for the reference square and in Table 5.2 for a ring-shaped physical domain, as a function of the number of subdomains $N$ and mesh size $1 / h$, for fixed $p=3, k=2, r=0$. Additional results for different regularity $k=0,1,2$ are plotted in Figures 5.1 and 5.2. The results show that the proposed preconditioner is scalable,

TABLE 5.1

$O A S$ preconditioner in $2 D$ parametric domain: condition number $\kappa_{2}\left(T_{O A S}\right)$ and iteration counts (it.) as a function of the number of subdomains $N$ and mesh size $1 / h$.

\begin{tabular}{|c|c|c|c|c|c|c|c|c|c|c|c|c|}
\hline & \multicolumn{12}{|c|}{ 2-level OAS prec. with $r=0, \quad p=3, k=2$ B-splines, square domain } \\
\hline \multirow[b]{2}{*}{$N$} & $1 / h$ & & $1 / h$ & 16 & $1 / h$ & $=32$ & $1 / h=$ & 64 & $1 / h=$ & 28 & $1 / h=$ & 256 \\
\hline & $\kappa_{2}$ & it. & $\kappa_{2}$ & it. & $\kappa_{2}$ & it & $\kappa_{2}$ & it. & $\kappa_{2}$ & it. & $\kappa_{2}$ & it. \\
\hline $2 \times 2$ & 6.64 & 13 & 6.30 & $\overline{12}$ & 6.57 & $\overline{12}$ & 10.13 & 15 & 17.86 & 18 & 33.45 & 23 \\
\hline $4 \times 4$ & & & 7.17 & 16 & 6.23 & 14 & 8.84 & 15 & 15.45 & 18 & 28.91 & 24 \\
\hline $8 \times 8$ & & & & & 7.52 & 17 & 6.14 & 14 & 9.54 & 16 & 17.08 & 19 \\
\hline $16 \times 16$ & & & & & & & 7.53 & 17 & 6.13 & 14 & 9.70 & 16 \\
\hline $32 \times 32$ & & & & & & & & & 7.03 & 16 & 6.13 & 14 \\
\hline $64 \times 64$ & & & & & & & & & & & 7.05 & 16 \\
\hline
\end{tabular}


TABLE 5.2

OAS preconditioner in $2 D$ physical domain: condition number $\kappa_{2}\left(T_{O A S}\right)$ and iteration counts (it.) as a function of the number of subdomains $N$ and mesh size $1 / h$ for 1 -level (upper table) and 2-level (lower table) OAS preconditioner.

\begin{tabular}{|c|c|c|c|c|c|c|c|c|c|c|}
\hline \multicolumn{11}{|c|}{ OAS prec. with $r=0, \quad p=3, k=2$ NURBS, ring domain } \\
\hline & $N$ & $\begin{array}{l}1 / h=8 \\
\kappa_{2} \quad \text { it. }\end{array}$ & \multicolumn{2}{|c|}{$1 / h=16$} & \multicolumn{2}{|c|}{$1 / h=32$} & \multicolumn{2}{|c|}{$1 / h=64$} & $\begin{array}{c}1 / h= \\
\kappa_{2}\end{array}$ & $\begin{array}{r}128 \\
\text { it. }\end{array}$ \\
\hline [2 & $2 \times 2$ & 7.69 & 13.07 & 17 & 25.10 & 21 & 49.49 & 30 & 98.47 & 41 \\
\hline 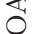 & $4 \times 4$ & & 18.54 & 22 & 39.42 & 29 & 81.28 & 41 & 165.02 & 58 \\
\hline 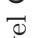 & $8 \times 8$ & & & & 65.75 & 38 & 146.45 & 54 & 307.67 & 78 \\
\hline 5 & $16 \times 16$ & & & & & & 255.98 & 73 & $5.75 \mathrm{e} 2$ & 106 \\
\hline I & $32 \times 32$ & & & & & & & & $1.02 \mathrm{e} 3$ & 146 \\
\hline 20 & $2 \times 2$ & 7.30 & 6.98 & 14 & 11.44 & 17 & 20.58 & 22 & 38.97 & 30 \\
\hline 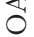 & $4 \times 4$ & & 8.12 & 18 & 10.62 & 20 & 19.60 & 23 & 37.72 & 32 \\
\hline 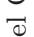 & $8 \times 8$ & & & & 8.41 & 19 & 13.92 & 21 & 29.88 & 27 \\
\hline อ & $16 \times 16$ & & & & & & 8.32 & 19 & 15.50 & 22 \\
\hline$\vec{N}$ & $32 \times 32$ & & & & & & & & 8.34 & 19 \\
\hline
\end{tabular}
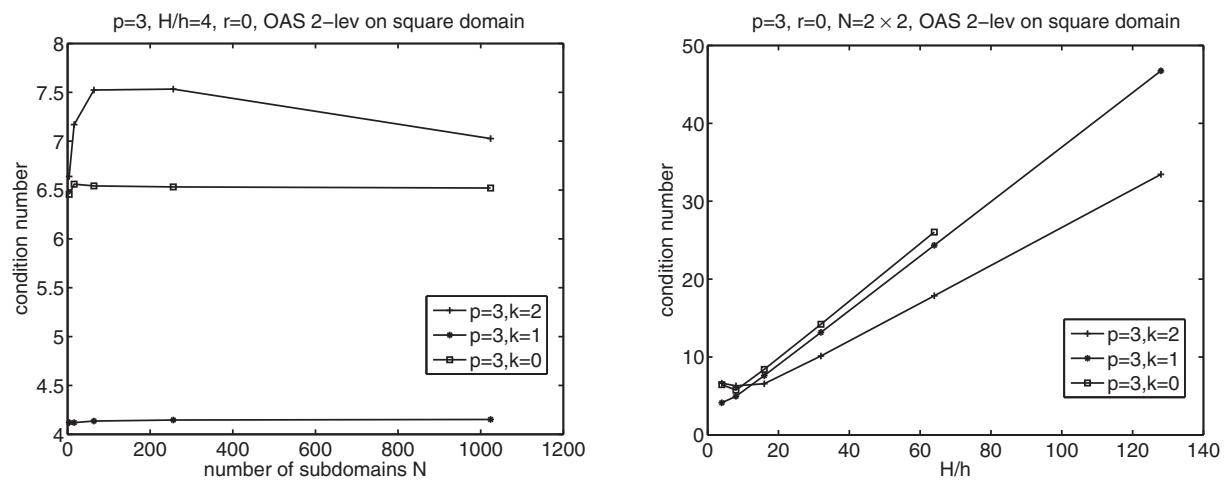

FIG. 5.1. Plot of $\kappa_{2}\left(T_{O A S}\right)$ as a function of $N$ (left) and $H / h$ (right) for $2 D$ parametric domain.
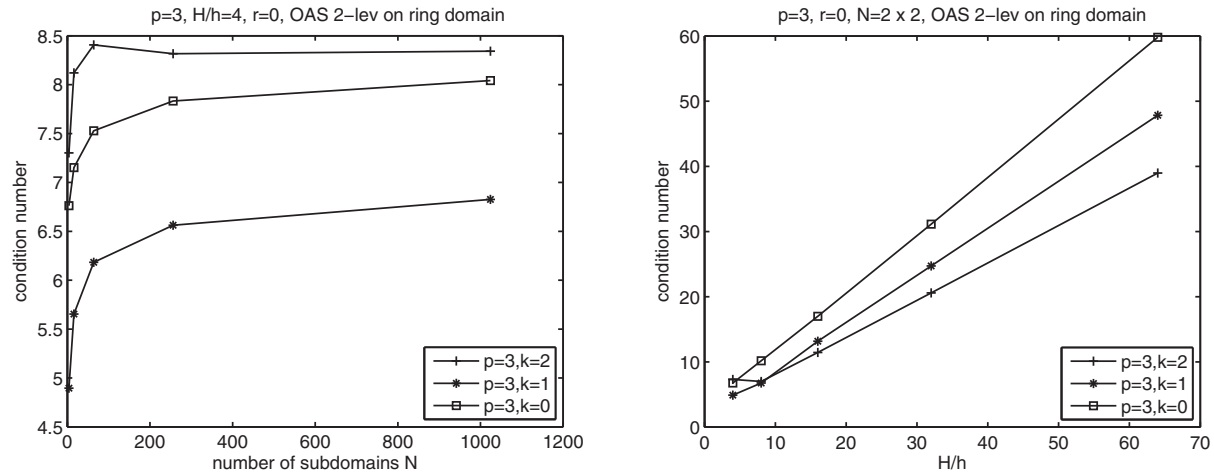

Fig. 5.2. Plot of $\kappa_{2}\left(T_{O A S}\right)$ as a function of $N$ (left) and $H / h$ (right) for $2 D$ ring domain.

since moving along the diagonal of the tables the condition number is bounded above by a constant independent of $N$. As expected, the coarse problem is essential for scalability, since the results of the first part of Table 5.2 show that the 1-level preconditioner (without coarse problem) has condition numbers growing with $N$ along the diagonals. The results of both tables and figures also confirm the main bound of 
TABLE 5.3

2-level OAS preconditioner in parametric domain: condition number $\kappa_{2}\left(T_{O A S}\right)$ and iteration counts (it.) as a function of the spline polynomial degree $p$ and the regularity $k$. Fixed $1 / h=64$, $N=4 \times 4, H / h=16$, symmetric minimal overlap $(r=0)$.

\begin{tabular}{|c|c|c|c|c|c|c|c|c|c|}
\hline \multicolumn{10}{|c|}{ Square domain, $h=1 / 64, N=4 \times 4,(H / h=16), r=0$} \\
\hline & \multirow[b]{2}{*}{$k$} & \multicolumn{2}{|c|}{$p=2$} & \multicolumn{2}{|c|}{$p=3$} & \multicolumn{2}{|c|}{$p=4$} & \multicolumn{2}{|c|}{$p=5$} \\
\hline & & $\kappa_{2}$ & it. & $\kappa_{2}$ & it. & $\kappa_{2}$ & it. & $\kappa_{2}$ & it. \\
\hline \multirow{5}{*}{$\begin{array}{l}0 \\
0 \\
0 \\
0 \\
0 \\
Z\end{array}$} & 0 & $2.22 \mathrm{e} 3$ & 187 & $4.30 \mathrm{e} 3$ & $\overline{252}$ & $7.04 \mathrm{e} 3$ & 307 & $1.09 \mathrm{e} 4$ & 375 \\
\hline & 1 & 311.56 & 71 & $1.01 \mathrm{e} 3$ & 126 & $2.18 \mathrm{e} 3$ & 182 & $3.30 \mathrm{e} 3$ & 222 \\
\hline & 2 & & & 327.21 & 72 & 842.40 & 116 & $1.46 \mathrm{e} 3$ & 148 \\
\hline & 3 & & & & & 381.73 & 76 & 734.29 & 106 \\
\hline & 4 & & & & & & & 445.91 & 82 \\
\hline \multirow{5}{*}{$\begin{array}{l}\frac{\pi}{4} \\
0 \\
0 \\
0 \\
0 \\
0 \\
\text { N }\end{array}$} & 0 & 8.91 & 18 & 8.52 & 17 & 8.52 & 17 & 8.68 & 17 \\
\hline & 1 & 9.69 & 16 & 8.53 & 15 & 7.01 & 14 & 6.13 & 13 \\
\hline & 2 & & & 8.90 & 15 & 9.44 & 19 & 23.05 & 23 \\
\hline & 3 & & & & & 6.19 & 12 & 9.52 & 18 \\
\hline & 4 & & & & & & & 15.75 & 18 \\
\hline
\end{tabular}

Theorem 3.1: moving along each table row, the condition numbers grow linearly with the increasing ratio $H / h$ (here for fixed overlap index $r=0$ we have $H / \gamma=O(H / h)$ ); moving along each table column, the condition number decreases about linearly for decreasing ratio $H / h$ (i.e., increasing $N$ for fixed $h$ ), except the last value of each column where $\kappa_{2}$ increases because the subdomains become so small that their excessive overlap causes a sudden jump of the maximum eigenvalue of $T_{O A S}$ (not reported).

5.2. 2D tests: OAS dependence on $\boldsymbol{p}$ and $\boldsymbol{k}$. The aim of this test is to compare the behavior of unpreconditioned conjugate gradient (CG) and 2-level OAS PCG with respect to the polynomial degree $p$ and spline regularity $k$. We recall that our theory does not cover the dependence of the 2-level OAS preconditioner on $p$ and $k$. The domain considered is the unit square discretized by a mesh of size $h=1 / 64$, subdivided into $N=4 \times 4$ subdomains with symmetric minimal overlap $r=0$. Indeed if $k$ is odd, we take two common functions instead of one between adjacent univariate subspaces in order to preserve symmetry in terms of basis functions. Thus, the number of common basis functions between adjacent univariate subspaces is $2 r+1+\bmod (k, 2)=1+\bmod (k, 2)$. Table 5.3 reports the condition numbers $\kappa_{2}$ and iteration counts it. varying $p$ from 2 to 5 and $k$ from 0 to 4 . The unpreconditioned CG condition numbers and iteration counts increase when $p$ grows, while they clearly improve when the regularity $k$ increases. The condition number of the 2-level OAS preconditioner seems to have a good behavior with respect to $k$ for fixed $p$, while the dependence on $p$ is less clear, since for $k=0$ and $k=1$ the condition number remains bounded when increasing $p$, but for $k=2$ and $k=3$ it grows. For the case $k=1, p=3$, we remark that earlier works by Bialecki et al. [13, 14] have studied Schwarz algorithms for orthogonal spline collocation with Hermite bicubics.

In order to investigate further the 2-level OAS preconditioner for higher values of $p$, we consider in Table 5.4 a discrete problem of smaller dimensions with $h=1 / 32$ and $N=2 \times 2$ subdomains. Now $p$ varies from 2 to 10 , and the regularity is either maximal $(k=p-1)$ or minimal $(k=0)$, while we increase the overlap $r$ from symmetric minimal $(r=0)$ to symmetric generous $(r=p)$. Thus, the number of common basis functions between adjacent univariate subspaces is again $2 r+1+\bmod (k, 2)$. The condition numbers reported in Table 5.4 show that unpreconditioned CG is strongly ill-conditioned for increasing $p$, while 2-level OAS performs much better. When the overlap is generous $(r=p)$ the 2-level OAS preconditioner becomes optimal with 
TABLE 5.4

2-level OAS preconditioner in parametric domain: condition number $\kappa_{2}\left(T_{O A S}\right)$ as a function of the spline polynomial degree $p$ for maximal regularity $k=p-1$ (left) and minimal regularity $k=0$ (right), with different levels of overlap from symmetric minimal $(r=0)$ to symmetric generous $(r=p)$. Fixed $1 / h=32, N=2 \times 2, H / h=16$.

\begin{tabular}{|c|c|c|c|c|c|c|c|c|}
\hline \multirow[b]{3}{*}{$p$} & \multicolumn{5}{|c|}{$k=p-1$} & \multicolumn{3}{|c|}{$k=0$} \\
\hline & \multirow[t]{2}{*}{ No prec. } & \multicolumn{4}{|c|}{ 2-level OAS } & \multirow[t]{2}{*}{ No prec. } & \multicolumn{2}{|c|}{ 2-level OAS } \\
\hline & & $r=0$ & $r=2$ & $r=4$ & $r=p$ & & $r=0$ & $r=p$ \\
\hline 2 & 78.12 & 7.08 & 4.63 & 4.11 & 4.63 & 554.89 & 8.98 & 4.87 \\
\hline 3 & 82.10 & 6.71 & 4.24 & 4.32 & 4.18 & $1.07 \mathrm{e}+3$ & 8.46 & 4.88 \\
\hline 4 & 206.71 & 6.02 & 4.10 & 4.29 & 4.29 & $1.76 \mathrm{e}+3$ & 8.47 & 4.92 \\
\hline 5 & $1.57 \mathrm{e}+3$ & 15.52 & 4.67 & 4.61 & 4.76 & $1.26 \mathrm{e}+4$ & 8.65 & 4.97 \\
\hline 6 & $1.29 \mathrm{e}+4$ & 12.64 & 4.88 & 4.66 & 4.79 & $1.53 \mathrm{e}+5$ & 8.80 & 4.98 \\
\hline 7 & $1.02 \mathrm{e}+5$ & 55.09 & 6.84 & 5.21 & 4.99 & $1.98 \mathrm{e}+6$ & 9.13 & 4.99 \\
\hline 8 & $2.99 \mathrm{e}+5$ & 37.43 & 7.61 & 5.35 & 4.98 & $1.86 \mathrm{e}+6$ & 10.55 & 4.98 \\
\hline 9 & $1.07 \mathrm{e}+6$ & 289.61 & 13.12 & 6.62 & 4.99 & $2.96 \mathrm{e}+6$ & 12.23 & 4.99 \\
\hline 10 & $1.24 \mathrm{e}+6$ & 156.85 & 13.44 & 6.20 & 4.99 & $6.34 \mathrm{e}+6$ & 13.48 & 4.99 \\
\hline
\end{tabular}

TABLE 5.5

2-level OAS preconditioner in a $3 D$ cubic domain: condition number $\kappa_{2}\left(T_{O A S}\right)$, extreme eigenvalues $\left(\lambda_{M A X}, \lambda_{\min }\right)$, and iteration counts (it.) as a function of the number of subdomains $N$. Fixed $H / h=4, p=3, k=2$.

\begin{tabular}{|c|c|c|c|c|}
\hline \multicolumn{5}{|c|}{ 2-level OAS, 3D cubic domain } \\
\hline & $r=0$ & & $r=1$ & \\
\hline$N$ & $\kappa_{2}=\lambda_{M A X} / \lambda_{\min }$ & it. & $\kappa_{2}=\lambda_{M A X} / \lambda_{\min }$ & it. \\
\hline $2 \times 2 \times 2$ & $18.60=8.20 / 0.44$ & 21 & $10.05=8.78 / 0.87$ & 19 \\
\hline $3 \times 3 \times 3$ & $18.80=8.26 / 0.44$ & 24 & $11.92=9.63 / 0.81$ & 21 \\
\hline $4 \times 4 \times 4$ & $19.66=8.29 / 0.42$ & 25 & $12.74=9.84 / 0.77$ & 22 \\
\hline $5 \times 5 \times 5$ & $19.46=8.30 / 0.43$ & 25 & $13.23=9.92 / 0.75$ & 23 \\
\hline $6 \times 6 \times 6$ & $19.52=8.31 / 0.43$ & 25 & $13.40=9.99 / 0.75$ & 23 \\
\hline
\end{tabular}

respect to $p$, as for standard spectral element discretizations, with condition number bounded by 5 . When the overlap is minimal $(r=0)$ or slightly larger $(r=2$ and $r=4$ ), the 2-level OAS preconditioner seems to be independent of $p$ up to $p=4$ or $p=5$, but the condition number starts to increase for higher values of $p$; for minimal regularity $(k=0)$, the growth is less than the standard $p^{2}$ growth for spectral elements, but for maximal regularity $(k=p-1)$ seems to be worse. We also remark that these results show that the performance of the 2-level OAS preconditioner improves when increasing the overlap size, in agreement with the bound of Theorem 3.1 and with the analogous results for finite and spectral elements.

5.3. 3D tests: OAS scalability in $N$. The condition number, extreme eigenvalues, and iteration counts of 2-level OAS PCG are reported in Table 5.5 for the reference cubic domain, as a function of the number of subdomains $N$ for fixed subdomain size $H / h=4$ (i.e., both $h$ and $H$ are decreasing proportionally as in a scaled speedup test), $p=3, k=2$, and overlap $r=0$ and $r=1$. These 3D results confirm that 2-level OAS is scalable, since the condition number is bounded from above by a constant independent of $N$. Moreover, the performance of the method improves when increasing the overlap size, since the minimal eigenvalue increases while the maximum eigenvalue remains bounded.

5.4. 2D and 3D tests: OAS robustness with respect to jump discontinuities. Finally, we investigate the robustness of our OAS preconditioners with respect to jumps discontinuities of the elliptic coefficient $\rho$. We consider two different 


central jump
\begin{tabular}{|l|l|l|l|}
\hline 1 & 1 & 1 & 1 \\
\hline 1 & $\rho$ & $\rho$ & 1 \\
\hline 1 & $\rho$ & $\rho$ & 1 \\
\hline 1 & 1 & 1 & 1 \\
\hline
\end{tabular}

(a)

\begin{tabular}{|c|c|c|c|}
\hline $10^{-3}$ & $10^{2}$ & $10^{-4}$ & $10^{2}$ \\
\hline $10^{1}$ & $10^{-1}$ & $10^{0}$ & $10^{4}$ \\
\hline $10^{-2}$ & $10^{3}$ & $10^{2}$ & $10^{-}$ \\
\hline $10^{0}$ & $10^{4}$ & $10^{-3}$ & $10^{1}$ \\
\hline
\end{tabular}

(b)

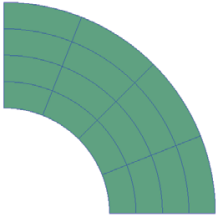

(c)

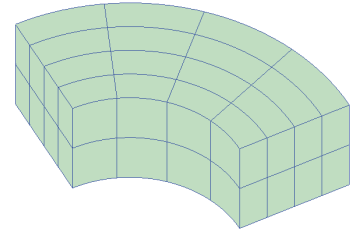

(d)

FIG. 5.3. (a) Central jump test with $4 \times 4$ subdomain decomposition of $\Omega$ with $\rho=10^{-4}, \ldots, 10^{4}$ in the $2 \times 2$ interior subdomains while $\rho=1$ in the boundary subdomains. (b) Random mix distribution of $\rho$ in the $4 \times 4$ subdomains. (c) $2 D$ ring domain. (d) $3 D$ ring domain.

TABLE 5.6

OAS robustness with respect to jump discontinuities in the elliptic coefficient $\rho$. Condition number $\kappa_{2}$, extreme eigenvalues $\lambda_{M A X}, \lambda_{\min }$, and iteration counts (it.) for central jump and random mix tests. Top table: $2 D$ ring domain with $h=1 / 64, N=4 \times 4, H / h=16$, overlap $r=1, p=3$, $k=2$. Bottom table: $3 D$ ring domain with fine mesh $16 \times 16 \times 8, N=4 \times 4 \times 2, H / h=4$, overlap $r=1, p=3, k=2$

\begin{tabular}{|c|c|c|c|c|c|c|c|}
\hline & & & $2 \mathrm{D}_{1}$ & g domain & & & \\
\hline & $\rho$ & $\begin{array}{r}\text { No prec. } \\
\kappa_{2}=\frac{\lambda_{M A X}}{\lambda_{\min }}\end{array}$ & it. & $\begin{array}{c}\text { 1-level OAS } \\
\kappa_{2}=\frac{\lambda_{M A X}}{\lambda_{\min }}\end{array}$ & it. & 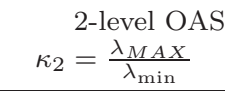 & it. \\
\hline द्वे & $10^{-4}$ & $4.53 e 6=\frac{12.43}{2.74 e-6}$ & 3840 & $82.89=\frac{4.00}{4.82 e-2}$ & 46 & $14.27=\frac{4.47}{3.13 e-1}$ & 27 \\
\hline$\Xi$ & $10^{-2}$ & $4.61 e 4=\frac{12.43}{2.70 e-4}$ & 996 & $83.19=\frac{4.00}{4.81 e-2}$ & 45 & $14.29=\frac{4.47}{3.13 e-1}$ & 26 \\
\hline 莺 & 1 & $2.29 e 3=\frac{13.60}{5.94 e-3}$ & 221 & $144.23=\frac{4.00}{2.77 e-2}$ & 64 & $19.54=\frac{4.47}{2.29 e-1}$ & 29 \\
\hline రี๊ & $10^{2}$ & $1.28 e 5=\frac{866.02}{6.77 e-3}$ & 1238 & $3.00 e 3=\frac{4.00}{1.33 e-3}$ & 62 & $15.16=\frac{4.48}{2.95 e-1}$ & 27 \\
\hline & $10^{4}$ & $1.27 e 7=\frac{8.63 e 4}{6.78 e-3}$ & 5565 & $2.80 e 5=\frac{4.00}{1.43 e-5}$ & 73 & $15.15=\frac{4.45}{2.94 e-1}$ & 31 \\
\hline & $m \operatorname{mix}$ & $2.90 e 9=\frac{8.5 e 5}{2.9 e-5}$ & $>10^{4}$ & $67=\frac{4}{5.9 e-2}$ & 20 & $7.94=\frac{4.17}{0.52}$ & 14 \\
\hline
\end{tabular}

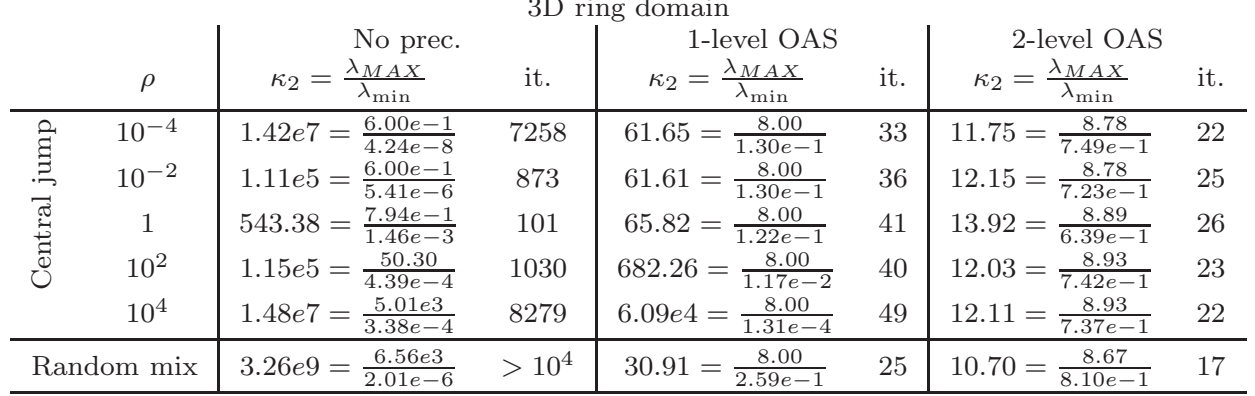

classical tests, which we call "central jump" and "random mix," in both a 2D quarter of ring decomposed in $4 \times 4$ subdomains and in a 3D "thick" quarter of ring decomposed into $4 \times 4 \times 2$ subdomains; see Figure 5.3. In the central jump test for the $2 \mathrm{D}$ ring, the elliptic coefficient $\rho$ varies of 8 orders of magnitude (from $10^{-4}$ to $10^{4}$ ) in the $2 \times 2$ central subdomains, while it is 1 in the surrounding subdomains.

In the random mix test, $\rho$ has random values varying 8 orders of magnitude among different subdomains. The $3 \mathrm{D}$ ring has the same $2 \mathrm{D}$ distributions of $\rho$ in its two layers of subdomains in the $z$-direction, except in the random mix test, where the second layer has the inverse of $\rho$ in the first layer. In the $2 \mathrm{D}$ test, we fix $h=1 / 64, H / h=16$, $p=3, k=2, r=1$, while in the 3D test the fine mesh consists of $16 \times 16 \times 8$ elements, $H / h=4, p=3, k=2, r=1$. Table 5.6 reports $\kappa_{2}$, extreme eigenvalues, 
iteration counts for unpreconditioned CG (left), 1-level OAS (middle), 2-level OAS (right), for both the 2D ring (top) and 3D ring (bottom). The results show clearly the robustness of 2-level OAS, with $\kappa_{2}$ and iterations independent of the jumps in $\rho$ in spite of the severe ill-conditioning (up to $O\left(10^{9}\right)$ ) of the unpreconditioned problem. 1level OAS performs well only when the jumps in $\rho$ decrease to 0 , while its convergence rate degenerates $\left(\lambda_{\min }\right.$ decays to zero) when the jumps increase. These results show that the robustness of overlapping Schwarz preconditioners with respect to elliptic coefficient discontinuities, known for finite and spectral element discretizations, holds also for our isogeometric preconditioner.

\section{REFERENCES}

[1] F. Auricchio, L. Beirão da Veiga, A. Buffa, C. Lovadina, A. Reali, and G. Sangalli, $A$ fully "locking-free" isogeometric approach for plane linear elasticity problems: A stream function formulation, Comput. Methods Appl. Mech. Engrg., 197 (2007), pp. 160-172.

[2] F. Auricchio, L. Beirão da Veiga, T.J.R. Hughes, A. Reali, and G. Sangalli, Isogeometric collocation methods, Math. Models Methods Appl. Sci., 20 (2010), pp. 2075-2107.

[3] F. Auricchio, L. Beirão da Veiga, C. Lovadina, and A. Reali, The importance of the exact satisfaction of the incompressibility constraint in nonlinear elasticity: Mixed FEMs versus NURBS-based approximations, Comput. Methods Appl. Mech. Engrg., 199 (2010), pp. 314-323.

[4] Y. Bazilevs, L. Beirão da Veiga, J.A. Cottrell, T.J.R. Hughes, and G. Sangalli, Isogeometric analysis: Approximation, stability and error estimates for h-refined meshes, Math. Models Methods Appl. Sci., 16 (2006), pp. 1-60.

[5] Y. Bazilevs, V.M. Calo, J.A. Cottrell, J.A. Evans, T.J.R. Hughes, S. Lipton, M.A. Scott, And T.W. Sederberg, Isogeometric analysis using T-splines, Comput. Methods Appl. Mech. Engrg., 199 (2010), pp. 229-263.

[6] Y. Bazilevs, V.M. Calo, J.A. Cottrell, T.J.R. Hughes, A. Reali, and G. Scovazzi, Variational multiscale residual-based turbulence modeling for large eddy simulation of incompressible flows, Comput. Methods Appl. Mech. Engrg., 197 (2007), pp. 173-201.

[7] Y. Bazilevs, V.M. Calo, T.J.R. Hughes, And Y. Zhang, Isogeometric fluid-structure interaction: Theory, algorithms, and computations, Comput. Mech., 43 (2008), pp. 3-37.

[8] L. Beirão da Veiga, A. Buffa, D. Cho, and G. Sangaldi, Isogeometric analysis using T-splines on two-patch geometries, Comput. Methods Appl. Mech. Engrg., 200 (2011), pp. $1787-1803$.

[9] L. Beirão Da Veiga, A. Buffa, J. Rivas, and G. Sangalli, Some estimates for $h-p-k$ refinement in isogeometric analysis, Numer. Math., 118 (2011), pp. 271-305.

[10] L. Beirão da Veiga, D. Cho, and G. Sangalli, Anisotropic NURBS approximation in isogeometric analysis, Comput. Methods Appl. Mech. Engrg., 209-212 (2012), pp. 1-11.

[11] D.J. Benson, Y. Bazilevs, E. De Luycker, M.C. Hsu, M. Scott, T.J.R. Hughes, and T. Belytschko, A generalized finite element formulation for arbitrary basis functions: From isogeometric analysis to XFEM, Internat. J. Numer. Methods Engrg., 83 (2010), pp. $765-785$.

[12] D.J. Benson, Y. Bazilevs, M.C. Hsu, and T.J.R. Hughes, Isogeometric shell analysis: The Reissner-Mindlin shell, Comput. Methods Appl. Mech. Engrg., 199 (2010), pp. 276-289.

[13] B. Bialecki, X.-C. Cai, M. Dryja, and G. Fairweather, An additive Schwarz algorithm for piecewise Hermite bicubic orthogonal spline collocation, in Domain Decomposition Methods in Science and Engineering, Contemp. Math. 157, AMS, Providence, RI, 1994, pp. 237244.

[14] B. Bialecki AND M. DRYjA, Multilevel additive and multiplicative methods for orthogonal spline collocation problems, Numer. Math., 77 (1997), pp. 35-58.

[15] A. Buffa, C. DE Falco, And G. SAngaldi, Isogeometric analysis: Stable elements for the $2 D$ Stokes equation, Internat. J. Numer. Methods Fluids, 65 (2011), pp. 1407-1422.

[16] A. Buffa, J. Rivas, G. Sangalli, and R. Vázquez, Isogeometric discrete differential forms in three dimensions, SIAM J. Numer. Anal., 49 (2011), pp. 818-844.

[17] A. Buffa, G. Sangalli, And R. VÁzquez, Isogeometric analysis in electromagnetics: B-splines approximation, Comput. Methods Appl. Mech. Engrg., 199 (2010), pp. 1143-1152.

[18] J.A. Cottrell, T.J.R. Hughes, And Y. Bazilevs, Isogeometric Analysis. Towards Integration of $C A D$ and FEA, Wiley, New York, 2009.

Copyright $@$ by SIAM. Unauthorized reproduction of this article is prohibited. 
[19] J.A. Cottrell, T.J.R. Hughes, and A. Reali, Studies of refinement and continuity in isogeometric structural analysis, Comput. Methods Appl. Mech. Engrg., 196 (2007), pp. 41604183.

[20] J.A. Cottrell, A. Reali, Y. Bazilevs, and T.J.R. Hughes, Isogeometric analysis of structural vibrations, Comput. Methods Appl. Mech. Engrg., 195 (2006), pp. 5257-5296.

[21] C. De Falco, A. Reali, and R. VazQuez, GeoPDEs: A research tool for isogeometric analysis of PDEs, Adv. Engrg. Software, 42 (2011), pp. 1020-1034.

[22] M. DRYJA AND O.B. WIDLund, Domain decomposition algorithms with small overlap, SIAM J. Sci. Comput., 15 (1994), pp. 604-620.

[23] J.A. Evans, Y. BAZILEvs, I. BABUŠKA, And T.J.R. Hughes, n-width, sup-infs, and optimality ratios for the k-version of the isogeometic finite element method, Comput. Methods Appl. Mech. Engrg., 198 (2009), pp. 1726-1741.

[24] G.E. FARIn, NURBS Curves and Surfaces: From Projective Geometry to Practical Use, A.K. Peters, Natick, MA, 1995.

[25] P.F. Fischer and J.W. LotTes, Hybrid Schwarz-multigrid methods for the spectral element method: Extensions to Navier-Stokes, J. Sci. Comput., 6 (2005), pp. 345-390.

[26] H. Gómez, V. Calo, Y. Bazilevs, and T.J.R. Hughes, Isogeometric analysis of the CahnHilliard phase-field model, Comput. Methods Appl. Mech. Engrg., 197 (2008), pp. 43334352.

[27] T.J.R. Hughes, J.A. Cottrell, and Y. Bazilevs, Isogeometric analysis: CAD, finite elements, NURBS, exact geometry, and mesh refinement, Comput. Methods Appl. Mech. Engrg., 194 (2005), pp. 4135-4195.

[28] T.J.R. Hughes, A. Reali, and G. Sangalli, Duality and unified analysis of discrete approximations in structural dynamics and wave propagation: Comparison of p-method finite elements with $k$-method NURBS, Comput. Methods Appl. Mech. Engrg., 197 (2008), pp. 4104-4124.

[29] T.J.R. Hughes, A. Reali, And G. Sangalli, Efficient quadrature for NURBS-based isogeometric analysis, Comput. Methods Appl. Mech. Engrg., 199 (2010), pp. 301-313.

[30] S. Lipton, J.A. Evans, Y. Bazilevs, T. Elguedu, and T.J.R. Hughes, Robustness of isogeometric structural discretizations under severe mesh distortion, Comput. Methods Appl. Mech. Engrg., 199 (2010), pp. 357-373.

[31] T. Mathew, Domain Decomposition Methods for the Numerical Solution of Partial Differential Equations, Springer, Berlin, 2008.

[32] L.F. PAVARINO And E. ZAMPIERI, Overlapping Schwarz and spectral element methods for linear elasticity and elastic waves, J. Sci. Comput., 27 (2006), pp. 51-73.

[33] L.F. Pavarino, E. Zampieri, R. Pasquetti, and F. Rapetti, Overlapping Schwarz methods for Fekete and Gauss-Lobatto spectral elements, SIAM J. Sci. Comput., 29 (2007), pp. 1073-1092.

[34] L. Piegl and W. Tiller, The NURBS Book, 2nd ed., Springer, New York, 1997.

[35] D.F. Rogers, An Introduction to NURBS with Historical Perspective. Academic Press, San Diego, CA, 2001.

[36] L.L. Schumaker, Spline Functions: Basic Theory, 3rd ed., Cambridge Mathematical Library, Cambridge University Press, Cambridge, UK, 2007.

[37] B.F. Smith, P. BJørstad, And W.D. Gropp, Domain Decomposition: Parallel Multilevel Methods for Elliptic Partial Differential Equations, Cambridge University Press, Cambridge, UK, 1996.

[38] A. Toselli And O.B. Widuund, Domain Decomposition Methods: Algorithms and Theory, Springer Ser. Comput. Math. 34, Springer, Berlin, 2004.

[39] W.A. Wall, M.A. Frenzel, and C. Cyron, Isogeometric structural shape optimization, Comput. Methods Appl. Mech. Engrg., 197 (2008), pp. 2976-2988.

[40] Y. Zhang, Y. Bazilevs, S. Goswami, C.L. BajaJ, and T.J.R. Hughes, Patient-specific vascular NURBS modeling for isogeometric analysis of blood flow, Comput. Methods Appl. Mech. Engrg., 196 (2007), pp. 2943-2959.

Copyright $@$ by SIAM. Unauthorized reproduction of this article is prohibited. 